\title{
INCREMENTAL GENERALIZED HOMOGENEITY, OBSERVER DESIGN AND SEMIGIOBAL STABILIZATION
}

\author{
Stefano Battilotti
}

\begin{abstract}
We introduce the notion of incremental generalized homogeneity, giving new results on semiglobal stabilization by output feedback and observer design and putting into a unifying framework the stabilization design for triangular (feedback/feedforward) and homogeneous systems. A state feedback controller and an asymptotic state observer are designed separately by dominating the generalized homogeneity degree of the nonlinearities with the degree of the linear approximation of the system and an output feedback controller is obtained according to a certainty-equivalence principle.
\end{abstract}

Key Words: Generalized homogeneity, state and output feedback, observers.

\section{INTRODUCTION}

Homogeneity and homogeneous approximations have been investigated by many authors for the analysis of the stability of an equilibrium point: [6], [5] and [13] to cite few. This theoretical setup has been exploited in the design of homogeneous controller/observers ([7], [8], [9], [1]): the idea is to design a stabilizing controller for the homogeneous approximation and asymptotic stability is preserved under any perturbation which does not change the homogeneous approximation.

In this paper we introduce the notion of generalized homogeneity (see the preliminary works [3] and [4]) and give conditions based on generalized homogeneity for semiglobal stabilization by output feedback. These conditions extend and improve conditions based on the notion of classical homogeneity ([7], [8], [9], [1]) in such a way that semiglobal stabilization by output feedback can be achieved for systems

$$
\begin{aligned}
& \dot{\mathbf{x}}=A \mathbf{x}+B \mathbf{u}+\phi(\mathbf{x}) \\
& \mathbf{y}=C \mathbf{x}+\psi(\mathbf{x})
\end{aligned}
$$

\footnotetext{
Manuscript received $i$ Article history $i$

S. Battilotti is with the Dipartimento di Ingegneria Informatica, Automatica e Gestionale "Antonio Ruberti"
}

with any function $\phi=\left(\begin{array}{lll}\phi_{1} & \cdots & \phi_{n}\end{array}\right)^{T}$ and $\psi$ such that for all $x=\left(\begin{array}{lll}x_{1} & \cdots & x_{n}\end{array}\right)^{T}$

$$
\begin{aligned}
& \left|\phi_{i}(x)\right| \leqslant\left(\sum_{j=1}^{i}\right) \Phi_{i j}\left(x_{1}, \ldots, x_{j}\right)\left|x_{j}\right|, i=1, \ldots, n, \\
& |\psi(x)| \leqslant \Psi_{1}\left(x_{1}\right)\left|x_{1}\right|
\end{aligned}
$$

(strict lower triangular $\phi$ ) or

$$
\left|\phi_{i}(x)\right| \leqslant\left(\sum_{j=i+2}^{n}\right) \Phi_{i j}\left(x_{i+2}, \ldots, x_{j}\right)\left|x_{j}\right|, i=1, \ldots, n,
$$$$
|\psi(x)| \leqslant\left(\sum_{j=2}^{n}\right) \Psi_{j}\left(x_{2}, \ldots, x_{j}\right)\left|x_{j}\right|, i=1, \ldots, n,
$$

(strict upper triangular $\phi$ ) for some continuous nonnegative functions $\Phi_{i j}$. While any such functions $\phi$ and $\psi$ are homogeneous in our generalized sense, additional restrictions must be assumed upon requiring homogeneity in the classical sense. Semiglobal stabilization by non-homogeneous output feedback has been proved for strict lower triangular $\phi$ in [14]. Under this regard and beyond [14], our result allows also for uncertain output terms like $y=c x$, where $c \in(0,1)$ is an unknown parameter, or saturated outputs as $y=$ $\operatorname{sat}(x)$. On the other hand, semiglobal stabilization by non-homogeneous output feedback has been proved for (non-strict) upper triangular $\phi$ in [2].

As to non-triangular $\phi$, our conditions based on generalized homogeneity extend and improve existing conditions based on the notion of classical homogeneity ([7], [8], [9], [1], [11], [10]) in such a way that one is 
able to cope with $\phi$ for which (consider as an example $n=3$ )

$$
\begin{aligned}
& \left|\phi_{1}\left(x_{1}, x_{2}, x_{3}\right)\right| \leqslant\left|x_{1}\right|^{\alpha_{1}}+\left|x_{1}\right|^{\beta_{1}}\left|x_{2}\right|^{\gamma_{1}}\left|x_{3}\right|^{\delta_{1}} \\
& \left|\phi_{2}\left(x_{1}, x_{2}, x_{3}\right)\right| \leqslant\left|x_{1}\right|^{\alpha_{2}}+\left|x_{1}\right|^{\beta_{2}}\left|x_{2}\right|^{\gamma_{2}}
\end{aligned}
$$

with no restrictions on $\phi_{3}$, where $\alpha_{j}, \beta_{j}, \gamma_{j}, j=1,2$, and $\delta_{1}$ are nonnegative reals such that either $0 \leqslant \delta_{1} \leqslant 1$ or $1<\delta_{1}<\frac{1-\gamma_{1}}{\gamma_{2}}$. Note that $\phi$ is not homogeneous (in the classical sense) if $\beta_{1}=3, \beta_{2}=1, \gamma_{1}=\frac{2}{3}$ and $\gamma_{2}=\frac{1}{4}$. Our result improve existing conditions for semiglobal stabilization by output feedback also with the introduction of the uncertain term $\psi$ in the output $y$.

In this paper a state feedback controller and an asymptotic state observer are designed separately by dominating the generalized homogeneity degree of $\phi$ and $\psi$ with the degree of the linear approximation of the system and an output feedback controller is obtained according to a certainty-equivalence principle. Our result puts into a unifying framework the stabilization problem for triangular (feedforward/feedback) and homogeneous systems. A novelty introduced by the notion of generalized homogeneity is the mixed low/high-gain observer/controller structure, in combination with saturated controls ([12]) and saturated state estimates ([14]). The saturation of the state estimates is a crucial issue of the observer design when $\phi$ and $\psi$ contain non-homogeneous terms.

\section{NOTATION}

- $\mathbb{R}^{n}$ (resp. $\mathbb{R}^{n \times n}$ ) is the set of $n$-dimensional real column vectors (resp. $n \times n$ matrices). $\mathbb{R}_{+}$(resp. $\mathbb{R}_{+}^{n}, \mathbb{R}_{+}^{n \times n}$ ) denotes the set of real non-negative numbers (resp. vectors in $\mathbb{R}^{n}$, matrices in $\mathbb{R}^{n \times n}$, with real non-negative entries). $\mathbb{R}_{>}$(resp. $\mathbb{R}_{>}^{n}$ ) denotes the set of real positive numbers (resp. vectors in $\mathbb{R}^{n}$ with real positive entries). For any $\mathfrak{c} \in \mathbb{R}$ set

$$
\mathbb{1}:=(1, \cdots, 1)^{T}, \mathbb{1} \cdot \mathfrak{c}:=(\mathfrak{c}, \cdots, \mathfrak{c})^{T} .
$$

- For any $G \in \mathbb{R}^{p \times n}$ we denote by $G_{i j}$ (or $[G]_{i j}$ ) the $(i, j)$-th entry of $G$ and by $G_{i}$ (or $[G]_{i}$ to avoid ambiguity) the $i$-th column of $G$. We retain a similar notation for functions.

- We denote by $\mathbf{C}^{j}(\mathscr{X}, \mathscr{Y})$, with $j \geqslant 0, \mathscr{X} \subset$ $\mathbb{R}^{n}$ and $\mathscr{Y} \subset \mathbb{R}^{p}$, the set of $j$-times continuously differentiable functions $f: \mathscr{X} \rightarrow \mathscr{Y}$. We denote by $\mathbf{D}^{j}\left(\mathbb{R}^{n}\right)$ the set of functions $f \in$ $\mathbf{C}^{j}\left(\mathbb{R}^{n}, \mathbb{R}^{n}\right)$ with decoupled components, viz. $f(x)=\left(f_{1}\left(x_{1}\right), \cdots, f_{n}\left(x_{n}\right)\right)^{T} . \mathbf{L}^{\infty}\left(\mathbb{R}_{+}, \mathbb{R}^{n}\right)$ is the set of functions $f \in \mathbf{C}^{0}\left(\mathbb{R}_{+}, \mathbb{R}^{n}\right)$ such that $\sup _{t \geqslant 0}\|f(t)\|<+\infty$ and $\mathbf{L}^{1}\left(\mathbb{R}_{+}, \mathbb{R}^{n}\right)$ is the set of $f \in \mathbf{C}^{0}\left(\mathbb{R}_{+}, \mathbb{R}^{n}\right)$ such that $\int_{0}^{\infty}\|f(\tau)\| d \tau<+\infty$.

- the increment $\Delta f$ of $f \in \mathbf{C}^{0}\left(\mathbb{R}^{n}, \mathbb{R}^{p}\right)$ from $x \in$ $\mathbb{R}^{n}$ to $y \in \mathbb{R}^{n}$ is defined as

$$
(\Delta f)(y, x):=f(y)-f(x)
$$

When we consider only increments of $x$ along the $l$-th coordinate $x_{l}, l=1, \ldots, n$, we define the increment $\Delta_{l} f$ of $f$ from $x \in \mathbb{R}^{n}$ to $y \in \mathbb{R}^{n}$ along the $l$-th direction as

$$
\begin{aligned}
& \left(\Delta_{l} f\right)(y, x) \\
& :=f\left(x_{1}, \ldots, x_{l-1}, y_{l}, x_{l+1}, \ldots, x_{n}\right)-f(x) .
\end{aligned}
$$

Set $\left(\Delta_{0} f\right)(x):=f(x)$.

- For any vectors $x, \varepsilon, \mathfrak{r} \in \mathbb{R}^{n}$, we define

$$
\begin{aligned}
\varepsilon^{\mathfrak{r}} & :=\left(\varepsilon_{1}^{\mathfrak{r}_{1}}, \cdots, \varepsilon_{n}^{\mathfrak{r}_{n}}\right)^{T}, \varepsilon^{\mathfrak{r}} \\
\diamond x & :=\left(\varepsilon_{1}^{\mathfrak{r}_{1}} x_{1}, \cdots, \varepsilon_{n}^{\mathfrak{r}_{n}} x_{n}\right)^{T},
\end{aligned}
$$

viz. the dilation of a vector $x$ with weight $\mathfrak{r}$. Moreover, for any vectors $x, y \in \mathbb{R}^{n}$ we write $x \leqslant$ $y$ (resp. $x<y, x=y$ ) if and only if $x_{i} \leqslant y_{i}$ (resp. $x_{i}<y_{i}, x_{i}=y_{i}$ ) for all $i=1, \ldots, n$. We retain a similar notation for pairs of vectors: we write $(x, y) \leqslant(z, w)(\operatorname{resp} .(x, y)=(z, w))$ if and only if $x_{i} \leqslant z_{i}$ and $y_{i} \leqslant w_{i}$ (resp. $x_{i}=z_{i}$ and $y_{i}=w_{i}$ ) for all $i=1, \ldots, n$.

\section{INCREMENTAL GENERALIZED HOMOGENEITY DEGREE}

\subsection{DEFINITIONS}

Below we introduce the notion of generalized homogeneity which generalizes along several directions the classical notion of homogeneity.

Definition III.1 (Incremental homogeneity in the generalized sense). A function $\phi \in \mathbf{C}^{0}\left(\mathbb{R}^{n}, \mathbb{R}\right)$ is said to be incrementally homogeneous in the generalized sense (i.g.h.) with triple $(\mathfrak{r}, \mathfrak{h}, \Phi)$ if there exist $\mathfrak{h} \in \mathbb{R}^{n}$, $\mathfrak{r} \in \mathbb{R}_{>}^{n}$ and $\Phi \in \mathbf{C}^{0}\left(\mathbb{R}^{2 n}, \mathbb{R}^{1 \times n}\right)$ such that

$$
(\Delta \phi)\left(\varepsilon^{\mathfrak{r}} \diamond y, \varepsilon^{\mathfrak{r}} \diamond x\right)=\left(\sum_{j=1}^{n}\right) \varepsilon^{\mathfrak{h}_{j}} \Phi_{j}(y, x)\left(y_{j}-x_{j} \chi 5\right)
$$

for all $\varepsilon>0$ and $x, y \in \mathbb{R}^{n}$. A function $\phi \in \mathbf{C}^{0}\left(\mathbb{R}^{n}, \mathbb{R}^{n}\right)$ is said to be incrementally homogeneous in the generalized sense (i.g.h.) with quadruple $(\mathfrak{r}, \mathfrak{d}, \mathfrak{h}, \Phi)$ if there exist $\mathfrak{d}, \mathfrak{h} \in \mathbb{R}^{n}, \mathfrak{r} \in \mathbb{R}_{>}^{n}$ and $\Phi \in \mathbf{C}^{0}\left(\mathbb{R}^{2 n}, \mathbb{R}^{n \times n}\right)$ such that

$$
\begin{aligned}
& \left(\Delta \phi_{i}\right)\left(\varepsilon^{\mathfrak{r}} \diamond y, \varepsilon^{\mathfrak{r}} \diamond x\right) \\
& \left.=\varepsilon^{\mathfrak{d}_{i}+\mathfrak{r}_{i}}\left(\sum_{j=1}^{n}\right) \varepsilon^{\mathfrak{h}_{j}} \Phi_{i j}(y, x)\left(y_{j}-x_{j}\right), i=1, \ldots, \text { k6 }\right)
\end{aligned}
$$

for all $\varepsilon>0$ and $x, y \in \mathbb{R}^{n}$. 
Note that if each increment $\Delta_{j} \phi$ (resp. $\Delta_{j} \phi_{i}$ ) from $x$ to $y$ is homogeneous in the classical sense with degree $\mathfrak{h}_{j}$ (resp. $\mathfrak{d}_{i}+\mathfrak{h}_{j}$ ) and weights $\mathfrak{r}$ then $\phi$ is i.g.h. with triple $(\mathfrak{r}, \mathfrak{h}, \Phi)$ (resp. with quadruple $(\mathfrak{r}, \mathfrak{d}, \mathfrak{h}, \Phi)$ ) for some function $\Phi$. This does not imply that $\Delta \phi$ is itself homogeneous in the classical sense.

The function $\phi(x):=x_{1}+x_{2}^{3}$ is i.g.h. with triple $(\mathfrak{r}, \mathfrak{h}, \Phi)$, where $\mathfrak{r}:=\left(\mathfrak{r}_{1}, \mathfrak{r}_{2}\right)^{T}, \mathfrak{h}:=\left(\mathfrak{r}_{1}, 3 \mathfrak{r}_{2}\right)^{T}$ and $\Phi(y, x)=\left(1, y_{2}^{2}+x_{2}^{2}+x_{2} y_{2}\right){ }^{*}$

Incremental homogeneity in the generalized sense give rise to the following definitions when the increment $\Delta \phi$ is calculated from $x \in \mathbb{R}^{n}$ to 0 .

Definition III.2 (Homogeneity in the generalized sense). A function $\phi \in \mathbf{C}^{0}\left(\mathbb{R}^{n}, \mathbb{R}\right)$ is said to be homogeneous in the generalized sense (g.h.) with triple $(\mathfrak{r}, \mathfrak{h}, \Phi)$ if there exist $\mathfrak{h} \in \mathbb{R}^{n}, \mathfrak{r} \in \mathbb{R}_{>}^{n}$ and $\Phi \in$ $\mathbf{C}^{0}\left(\mathbb{R}^{n}, \mathbb{R}^{1 \times n}\right)$ such that

$$
\phi\left(\varepsilon^{\mathfrak{r}} \diamond x\right)=\left(\sum_{j=1}^{n}\right) \varepsilon^{\mathfrak{h}_{j}} \Phi_{j}(x) x_{j}
$$

for all $\varepsilon>0$ and $x \in \mathbb{R}^{n}$. A function $\phi \in \mathbf{C}^{0}\left(\mathbb{R}^{n}, \mathbb{R}^{n}\right)$ is said to be homogeneous in the generalized sense (g.h.) with quadruple $(\mathfrak{r}, \mathfrak{d}, \mathfrak{h}, \Phi)$ if there exist $\mathfrak{d}, \mathfrak{h} \in \mathbb{R}^{n}$, $\mathfrak{r} \in \mathbb{R}_{>}^{n}$ and $\Phi \in \mathbf{C}^{0}\left(\mathbb{R}^{n}, \mathbb{R}^{n \times n}\right)$ such that

$\phi_{i}\left(\varepsilon^{\mathfrak{r}} \diamond x\right)=\varepsilon^{\mathfrak{d}_{i}+\mathfrak{r}_{i}}\left(\sum_{j=1}^{n}\right) \varepsilon^{\mathfrak{h}_{j}} \Phi_{i j}(x) x_{j}, i=1, \ldots, n(9)$

for all $\varepsilon>0$ and $x \in \mathbb{R}^{n}$.

I.g.h. implies g.h. and g.h. generalizes the classical notion of homogeneity.

There are functions, like $\sin x$, which are not i.g.h. but their absolute value is bounded by the absolute value of a function which is i.g.h. In many situations it is more convenient to have a homogeneous (in the generalized sense) upper bound. This motivates the following definitions.

Definition III.3 (Incremental homogeneity in the upper bound in the generalized sense). A function $\phi \in \mathbf{C}^{0}\left(\mathbb{R}^{n}, \mathbb{R}\right)$ is said to be incrementally homogeneous in the upper bound in the generalized sense

\footnotetext{
* The functions $\Phi$ can be readily calculated as

$\Phi_{i j}(y, x)$$$
:=\frac{\left(\Delta_{j} \phi_{i}\right)\left(\varepsilon^{\mathfrak{r}} \diamond y, \varepsilon^{\mathfrak{r}_{1}} y_{1}, \ldots, \varepsilon^{\mathfrak{r}_{j-1}} y_{j-1}, \varepsilon^{\mathfrak{r}_{j}} x_{j}, \ldots, \varepsilon^{\mathfrak{r}_{n}} x_{n}\right)}{\varepsilon^{\mathfrak{d}_{i}+\mathfrak{r}_{i}+\mathfrak{h}_{j}}\left(y_{j}-x_{j}\right)}
$$

as long as $\mathfrak{d}_{i}$ and $\mathfrak{h}_{j}$ can be selected in such a way that the righthand function in (7) is independent of $\varepsilon$. This is the case when $\phi_{i}$ is a polynomial function of its arguments. (i.g.h.b.) with triple $(\mathfrak{r}, \mathfrak{h}, \Phi)$ if there exist $\mathfrak{h} \in \mathbb{R}^{n}, \mathfrak{r} \in$ $\mathbb{R}_{>}^{n}$ and $\Phi \in \mathbf{C}^{0}\left(\mathbb{R}^{2 n}, \mathbb{R}_{+}^{n}\right)$ such that

$\left|(\Delta \phi)\left(\varepsilon^{\mathfrak{r}} \diamond y, \varepsilon^{\mathfrak{r}} \diamond x\right)\right| \leqslant\left(\sum_{j=1}^{n}\right) \varepsilon^{\mathfrak{h}_{j}} \Phi_{j}(y, x) \mid y_{j}-x_{j}(10)$

for all $\varepsilon \geqslant \varepsilon_{0}$ and $x, y \in \mathbb{R}^{n}$. A function $\phi \in$ $\mathbf{C}^{0}\left(\mathbb{R}^{n}, \mathbb{R}^{n}\right)$ is said to be incrementally homogeneous in the upper bound in the generalized sense (i.g.h.b.) with quadruple $(\mathfrak{r}, \mathfrak{d}, \mathfrak{h}, \Phi)$ if there exist $\mathfrak{d}, \mathfrak{h} \in \mathbb{R}^{n}, \mathfrak{r} \in \mathbb{R}_{>}^{n}$, $\Phi \in \mathbf{C}^{0}\left(\mathbb{R}^{2 n}, \mathbb{R}_{+}^{n \times n}\right)$ and $\varepsilon_{0}>0$ such that

$\left|\left(\Delta \phi_{i}\right)\left(\varepsilon^{\mathfrak{r}} \diamond y, \varepsilon^{\mathfrak{r}} \diamond x\right)\right|$

$\leqslant \varepsilon^{\mathfrak{d}_{i}+\mathfrak{r}_{i}}\left(\sum_{j=1}^{n}\right) \varepsilon^{\mathfrak{h}_{j}} \Phi_{i j}(y, x)\left|y_{j}-x_{j}\right|, i=1, \ldots$,

for all $\varepsilon \geqslant \varepsilon_{0}$ and $y, x \in \mathbb{R}^{n}$.

The function $\phi(x):=\sin x$ is i.g.h.u.b. with quadruple $(\mathfrak{r}, 0,0,1)$ but it has no i.g.h. degree. The function $\phi(x):=\left(x_{2}, x_{2}+x_{2}^{p}\right)^{T}, p \geqslant 1$, is i.g.h.u.b. with triple $(\mathfrak{r}, \mathfrak{d}, \mathfrak{h}, \Phi)$, where

$$
\begin{aligned}
& \mathfrak{r}:=\left(\begin{array}{l}
\mathfrak{r}_{1} \\
\mathfrak{r}_{2}
\end{array}\right), \mathfrak{d}=\left(\begin{array}{c}
\mathfrak{r}_{2}-\mathfrak{r}_{1} \\
\mathfrak{r}_{2}(p-1)
\end{array}\right), \mathfrak{h}=\left(\begin{array}{l}
0 \\
0
\end{array}\right), \\
& \Phi(y, x):=\left(\begin{array}{ll}
0 & 1 \\
0 & 1+\sum_{j=0}^{p-1}\left|x_{2}\right|^{p-j-1}\left|y_{2}\right|^{j}
\end{array}\right)
\end{aligned}
$$

for all $p \geqslant 1$.

Without loss of generality one can assume $\varepsilon_{0}=$ 1 , otherwise rescale $x$ and $y$ as $z=\varepsilon_{0}^{\mathfrak{r}} \diamond x$ and, respectively, $w=\varepsilon_{0}^{\mathfrak{r}} \diamond y$ and define new functions $\left[\Phi^{\prime}\right]_{j}(w, z):=\varepsilon_{0}^{\mathfrak{d}_{i}+\mathfrak{r}_{i}-\mathfrak{r}_{j}+\mathfrak{h}_{j}} \Phi_{j}\left(\varepsilon_{0}^{-\mathfrak{r}} \diamond w, \varepsilon_{0}^{-\mathfrak{r}} \diamond z\right)$ (resp. $\left.\left[\Phi^{\prime}\right]_{i j}(w, z):=\varepsilon_{0}^{\mathfrak{d}_{i}+\mathfrak{r}_{i}-\mathfrak{r}_{j}+\mathfrak{h}_{j}} \Phi_{i j}\left(\varepsilon_{0}^{-\mathfrak{r}} \diamond w, \varepsilon_{0}^{-\mathfrak{r}} \diamond z\right)\right)$.

The function $\phi=\left(\begin{array}{c}x_{2} \\ x_{2}^{2} \sin x_{1}\end{array}\right)$ is i.g.h.u.b. with triple $(\mathfrak{r}, \mathfrak{d}, \mathfrak{h}, \Phi)$, where

$$
\begin{aligned}
& \mathfrak{r}:=\left(\begin{array}{l}
\mathfrak{r}_{1} \\
\mathfrak{r}_{2}
\end{array}\right), \mathfrak{d}=\left(\begin{array}{l}
0 \\
0
\end{array}\right), \mathfrak{h}=\left(\begin{array}{c}
\mathfrak{r}_{2}-\mathfrak{r}_{1} \\
\mathfrak{r}_{2}+\mathfrak{r}_{1}
\end{array}\right), \\
& \Phi(y, x):=\left(\begin{array}{cc}
0 & 1 \\
x_{2}^{2} \frac{\left|\sin y_{1}-\sin x_{1}\right|}{\left|y_{1}-x_{1}\right|} & \left|y_{2}+x_{2}\right|
\end{array}\right) .
\end{aligned}
$$

$\dagger$

\footnotetext{
${ }^{\dagger}$ For each $i, j=1, \ldots, n$ the functions $\Phi_{i j}$ in (11) are obtained as long as $\mathfrak{d}_{i}$ and $\mathfrak{h}_{j}$ can be selected in such a way that$$
\sup _{\varepsilon \geqslant 1} \frac{\left|\left(\Delta_{j} \phi_{i}\right)\left(\varepsilon^{\mathfrak{r}} \diamond y, \varepsilon^{\mathfrak{r}_{1}} y_{1}, \ldots, \varepsilon^{\mathfrak{r}_{j-1}} y_{j-1}, \varepsilon^{\mathfrak{r}_{j}} x_{j}, \ldots, \varepsilon^{\mathfrak{r}_{n}} x_{n}\right)\right|}{\varepsilon^{\mathfrak{d}_{i}+\mathfrak{r}_{i}+\mathfrak{h}_{j}}\left|y_{j}-x_{j}\right|}
$$$$
\leqslant \Phi_{i j}(y, x)
$$

for all $x \in \mathbb{R}^{n}$ and $y \in \mathbb{R}$. This is the case when

$$
\frac{\left|\Delta_{j} \phi_{i}\left(y, y_{1}, \ldots, y_{j-1}, x_{j}, \ldots, x_{n}\right)\right|}{\left|y_{j}-x_{j}\right|}
$$

is bounded by a polynomial function of $x$ and $y$.
} 


\subsection{SOME RELATED PROPERTIES}

Throughout the paper we assume $\varepsilon_{0} \geqslant 1$ in the above definitions. We discuss only few properties of incremental homogeneity (in the generalized sense) which are extensively used throughout the paper.

(P1) (mixing property) Any i.g.h.u.b. function $\phi \in$ $\mathbf{C}^{0}\left(\mathbb{R}^{n}, \mathbb{R}^{n}\right)$ with quadruple $(\mathfrak{r}, \mathfrak{d}, \mathfrak{h}, \Phi)$ and diagonal $\Phi$ is also i.g.h.u.b. with quadruple $\left(\mathfrak{r}, \mathfrak{d}^{\prime}, \mathfrak{h}^{\prime}, \Phi\right)$ for all pairs $\left(\mathfrak{d}^{\prime}, \mathfrak{h}^{\prime}\right)$ such that $\mathfrak{d}^{\prime}+\mathfrak{h}^{\prime} \geqslant \mathfrak{d}+\mathfrak{h}$.

In particular, we can swap the degrees $(\mathfrak{d}, \mathfrak{h})$ as $\left(\mathfrak{d}^{\prime}, \mathfrak{h}^{\prime}\right)=(\mathfrak{h}, \mathfrak{d})$ or replace $(\mathfrak{d}, \mathfrak{h})$ with their arithmetic means $\left(\mathfrak{d}^{\prime}, \mathfrak{h}^{\prime}\right)=\left(\frac{\mathfrak{d}+\mathfrak{h}}{2}, \frac{\mathfrak{d}+\mathfrak{h}}{2}\right)$.

When composing two functions the i.g.h.u.b. degree behaves as follows.

(P2) (chaining rule) For any i.g.h.u.b. functions $\phi \in \mathbf{C}^{0}\left(\mathbb{R}^{n}, \mathbb{R}^{n}\right)$ with quadruple $(\mathfrak{r}, \mathfrak{d}, \mathfrak{h}, \Phi)$ and $\psi \in$ $\mathbf{C}^{0}\left(\mathbb{R}^{n}, \mathbb{R}^{n}\right)$ with quadruple $(\mathfrak{r},-\mathfrak{h}, \mathfrak{p}, \Psi)$ if there exists $\Phi^{0} \in \mathbf{C}^{0}\left(\mathbb{R}^{2 n}, \mathbb{R}_{+}^{n \times n}\right)$ such that for each $i, j=1, \ldots, n$ and for all $y, x \in \mathbb{R}^{n}$

$\sup _{\varepsilon \geqslant 1} \Phi_{i j}\left(\varepsilon^{-\mathfrak{r}} \diamond \psi\left(\varepsilon^{\mathfrak{r}} \diamond y\right), \varepsilon^{-\mathfrak{r}} \diamond \psi\left(\varepsilon^{\mathfrak{r}} \diamond x\right)\right) \leqslant \Phi_{i j}^{0}(y, x)$

then $\phi \circ \psi$ is i.g.h.u.b. with quadruple $\left(\mathfrak{r}, \mathfrak{d}, \mathfrak{p}, \Phi^{0} \Psi\right)$. Indeed, for all $\varepsilon \geqslant 1$ and $y, x \in \mathbb{R}^{n}$

$$
\begin{aligned}
& \left|\left(\Delta\left(\phi_{i} \diamond \psi\right)\right)\left(\varepsilon^{\mathfrak{r}} \diamond y, \varepsilon^{\mathfrak{r}} \diamond x\right)\right|= \\
& \left|\left(\Delta \phi_{i}\right)\left(\varepsilon^{\mathfrak{r}} \diamond\left(\varepsilon^{-\mathfrak{r}} \diamond \psi\left(\varepsilon^{\mathfrak{r}} \diamond y\right)\right), \varepsilon^{\mathfrak{r}} \diamond\left(\varepsilon^{-\mathfrak{r}} \diamond \psi\left(\varepsilon^{\mathfrak{r}} \diamond x\right)\right)\right)\right| \\
& \leqslant \varepsilon^{\mathfrak{r}_{i}+\mathfrak{o}_{i}}\left(\sum_{j=1}^{n}\right) \varepsilon^{\mathfrak{p}_{j}}\left[\Phi^{0} \Psi\right] i j(y, x)\left|y_{j}-x_{j}\right|, \\
& i=1, \ldots, n .
\end{aligned}
$$

After shifting a vector of functions or the vector of its arguments the i.g.h.u.b. degree of the vector function transforms as follows.

(P3) (shifting rules) Let $(A, B)$ be in Brunowski canonical form. Note that $A^{T}$ is the Moore-Penrose pseudoinverse of $A$, viz. $A^{T} A A^{T}=A^{T}, A A^{T} A=$ $A, \quad\left(A^{T} A\right)^{T}=A^{T} A$ and $\left(A A^{T}\right)^{T}=A A^{T}$. Therefore $I-A A^{T}=B B^{T}$ is the orthogonal projection onto $(\operatorname{Im}\{A\})^{\perp}=\operatorname{Im}\left\{I-A A^{T}\right\}$ while $I-A^{T} A$ is the orthogonal projection onto $\left(\operatorname{Im}\left\{A^{T}\right\}\right)^{\perp}=\operatorname{Im}\{I-$ $\left.A^{T} A\right\}(\operatorname{Im}\{W\}$ denotes the vector space generated by the columns of the matrix $W$ ). It is easy to see that

(P3.1)for any $\mathfrak{w} \in \operatorname{Im}\left\{I-A A^{T}\right\} \quad$ (resp. $\mathfrak{w} \in \operatorname{Im}\{I-$ $\left.\left.A^{T} A\right\}\right)$ and i.g.h.u.b. $\phi \in \mathbf{C}^{0}\left(\mathbb{R}^{n}, \mathbb{R}^{n}\right)$ with quadruple $(\mathfrak{r}, \mathfrak{d}, \mathfrak{h}, \Phi), A \phi$ (resp. $\left.A^{T} \phi\right)$ is i.g.h.u.b. with quadruple $(\mathfrak{r}, A(\mathfrak{d}+\mathfrak{r})-\mathfrak{r}+\mathfrak{w}, \mathfrak{h}, A \Phi)\left(\operatorname{resp} .\left(\mathfrak{r}, A^{T}(\mathfrak{d}+\mathfrak{r})-\right.\right.$ $\left.\left.\mathfrak{r}+\mathfrak{w}, \mathfrak{h}, A^{T} \Phi\right)\right)$.

Indeed, for all $\varepsilon \geqslant 1$ and $y, x \in \mathbb{R}^{n}$ and $\mathfrak{w} \in$ $\operatorname{Im}\left\{I-A A^{T}\right\}$

$$
\begin{aligned}
& \left|\left(\Delta[A \phi]_{i}\right)\left(\varepsilon^{\mathfrak{r}} \diamond y, \varepsilon^{\mathfrak{r}} \diamond x\right)\right|=\left|\left(\Delta \phi_{i+1}\right)\left(\varepsilon^{\mathfrak{r}} \diamond y, \varepsilon^{\mathfrak{r}} \diamond x\right)\right| \\
& \leqslant \varepsilon^{\mathfrak{r}_{i}+[A(\mathfrak{d}+\mathfrak{r})-\mathfrak{r}+\mathfrak{w}]_{i}}\left(\sum_{l=1}^{n}\right) \varepsilon^{\mathfrak{h}_{l}}[A \Phi]_{i l}(y, x)\left|y_{l}-x_{l}\right|, \\
& i=1, \ldots, n-1 .
\end{aligned}
$$

The proof for $A^{T} \phi$ goes likewise.

(P3.2) for any $\mathfrak{w} \in \operatorname{Im}\left\{I-A^{T} A\right\}$ (resp. $\mathfrak{w} \in \operatorname{Im}\{I-$ $\left.\left.A A^{T}\right\}\right)$ and i.g.h.u.b. $\phi \in \mathbf{C}^{0}\left(\mathbb{R}^{n}, \mathbb{R}^{n}\right)$ with quadruple $(\mathfrak{r}, \mathfrak{d}, \mathfrak{h}, \Phi)$ and constant $\Phi, \phi \circ A$ (resp. $\left.\phi \circ A^{T}\right)$ is i.g.h.u.b. with quadruple $\left(\mathfrak{r}, \mathfrak{d}, A^{T}(\mathfrak{h}-\mathfrak{r})+\mathfrak{r}+\mathfrak{w}, \Phi A\right)$ (resp. $\left.\left(\mathfrak{r}, \mathfrak{d}, A(\mathfrak{h}-\mathfrak{r})+\mathfrak{r}+\mathfrak{w}, \Phi A^{T}\right)\right)$. Indeed, for all $\varepsilon \geqslant 1$, and $y, x \in \mathbb{R}^{n}$ and $\mathfrak{w} \in \operatorname{Im}\left\{I-A^{T} A\right\}$

$$
\begin{aligned}
& \left|\left(\Delta[\phi \circ A]_{i}\right)\left(\varepsilon^{\mathfrak{r}} \diamond y, \varepsilon^{\mathfrak{r}} \diamond x\right)\right|= \\
& \left|\left(\Delta \phi_{i}\right)\left(\varepsilon^{\mathfrak{r}} \diamond\left(\varepsilon^{-\mathfrak{r}} \diamond A\left(\varepsilon^{\mathfrak{r}} \diamond y\right)\right), \varepsilon^{\mathfrak{r}} \diamond\left(\varepsilon^{-\mathfrak{r}} \diamond A\left(\varepsilon^{\mathfrak{r}} \diamond x\right)\right)\right)\right| \\
& \leqslant \varepsilon^{\mathfrak{r}_{i}+\mathfrak{d}_{i}}\left(\sum_{l=1}^{n}\right) \varepsilon^{\left[A^{T}(\mathfrak{h}-\mathfrak{r})+\mathfrak{r}+\mathfrak{w}\right]_{l}}[\Phi A]_{i l}\left|y_{l}-x_{l}\right|, \\
& i=1, \ldots, n .
\end{aligned}
$$

The proof for $\phi \circ A^{T}$ goes in the same way.

\section{STATE FEEDBACK STABILIZATION}

\subsection{Statement of the main result}

Consider the system

$$
\dot{\mathbf{x}}=A \mathbf{x}+B \mathbf{u}+\phi(\mathbf{x}),
$$

with state $x \in \mathbb{R}^{n}$, input $u \in \mathbb{R},(A, B)$ a Brunowski canonical form and $\phi \in \mathbf{C}^{0}\left(\mathbb{R}^{n}, \mathbb{R}^{n}\right), \quad \phi(0)=0$. Throughout the paper we use the notation $\mathbf{x}, \mathbf{u}$ for the functions of time and $x, u$ for their values.

The main result of this section states that if $\phi$ is i.g.h.u.b. with weights and degrees properly related then the equilibrium $x=0$ of (14) can be semiglobally stabilized by linear state feedback controller. The class of stabilizing controllers has been deliberately restricted to be linear since this is quite natural in the context of semiglobal stabilization by state feedback. On the other hand, genuinely nonlinear controllers are more natural in the context of global stabilization which is not the aim of this paper. The reader should also put our stabilization result in perspective with the observer design result of the next section (theorem V.1) for obtaining a certainty equivalence principle for semiglobal stabilization by output feedback which is the final aim of this paper (theorem VI.1). The resulting class of stabilizing output feedback controllers is the composition of linear dynamic controllers with static nonlinearities, which is enough general as far as semiglobal stabilization is concerned.

Theorem IV.1 Assume that

(S) (incremental homogeneity) $\phi \in \mathbf{C}^{0}\left(\mathbb{R}^{n}, \mathbb{R}^{n}\right)$ is i.g.h.u.b. with quadruple $(\mathfrak{r}, \mathfrak{f}, \hat{\mathfrak{f}}, \Phi)$ with $\Phi(0,0)=0$ and $\hat{\mathfrak{f}}_{1}:=\mathfrak{f}_{1}, \hat{\mathfrak{f}}_{j+1}:=\mathfrak{r}_{j+1}-\mathfrak{r}_{j}-\mathfrak{f}_{j}, j=1, \ldots, n-1$, such that $\mathfrak{f}_{j} \leqslant \hat{\mathfrak{f}}_{j+1} \leqslant \mathfrak{f}_{j+1}, j=1, \ldots, n-1$. 
For each compact set $\mathscr{R} \subset \mathbb{R}^{n}$ around the origin, there exist a positive definite diagonal $\Gamma_{s} \in \mathbb{R}^{n \times n}$ and $\varepsilon \geqslant 1$ such that

$$
\begin{aligned}
& \mathbf{u}=F(\mathbf{x}):=B^{T} F^{(n)}(\mathbf{x}) \\
& F^{(i)}(x):=-\varepsilon^{2 \mathfrak{f}} \diamond\left\{\Gamma_{s}\left[x-A^{T} F^{(i-1)}(x)\right]\right\}, \\
& i=1, \ldots, n, F^{(0)}(x):=0,
\end{aligned}
$$

asymptotically stabilizes the equilibrium $\mathbf{x}=0$ of (14) with region of attraction containing $\mathscr{R}$.

Remark IV.1 Assumption $\Phi(0,0)=0$ is the simplest one which guarantees that the linear approximation of (14) around the origin is stabilizable. The minimal requirement for this is

$-2 \Gamma_{s}+\left[I-A^{T} \Gamma_{s}\right]^{-1}\left[A+A^{T} \Gamma_{s}^{2}+\Phi(0,0)\left(I+A^{T} \Gamma_{s}\right)\right]$

$+\left[A+A^{T} \Gamma_{s}^{2}+\Phi(0,0)\left(I+A^{T} \Gamma_{s}\right)\right]^{T}\left[I-A^{T} \Gamma_{s}\right]^{-T}<0$

which is always satisfied by some positive definite diagonal $\Gamma_{s}$ for either strict lower triangular or strict upper triangular of $\Phi(0,0)$. $\square$

Remark IV.2 The controller (16) is homogeneous (in the classical sense) only if $\mathfrak{f}_{j}=\mathfrak{f}_{0}$ for all $j=1, \ldots, n$, which corresponds to the choice $\mathfrak{r}_{j+1}-\mathfrak{r}_{j}=2 \mathfrak{f}_{0}$ in (S). In other words the gaps between each pair of consecutive weights are all equal to $2 \mathfrak{f}_{0}$. Under this restriction, (S) simply says that $\phi$ is homogeneous (in the upper bound) with degree $2 \mathfrak{f}_{0}$ and weights $\mathfrak{r}$. $\square$

\subsection{CONSTRUCTIVE PROCEDURE FOR THE CONTROLLER (16)}

Given the desired region of attraction $\mathscr{R} \subset \mathbb{R}^{n}$, the construction of the state feedback controller (16) is accomplished according to the following steps:

(I) find a diagonal positive definite $\Gamma_{s} \in \mathbb{R}^{n \times n}$ such that $\ddagger$

\footnotetext{
${ }^{\ddagger}$ Inequality (18) can be always solved for some diagonal positive definite $\Gamma_{s} \in \mathbb{R}^{n \times n}$ since $\mathfrak{T}$ can be defined recursively as $\mathfrak{T}=\mathfrak{T}^{(n)}$ where

$$
\begin{aligned}
& \mathfrak{T}^{(j)}:=\left[\begin{array}{c|c}
\mathfrak{T}^{(j-1)} & \mathfrak{N}^{(j-1)} \\
\left.\hline \mathfrak{N}^{(j-1)}\right)^{T} & -2\left(\left[\Gamma_{s}\right]_{j j}-\left[\Gamma_{s}\right]_{j-1, j-1}\right)
\end{array}\right], \\
& \mathfrak{T}^{(1)}:=-2\left[\Gamma_{s}\right]_{11},
\end{aligned}
$$

and $\mathfrak{N}^{(j-1)} \in \mathbf{C}^{0}\left(\mathbb{R}^{j-1}, \mathbb{R}^{j-1}\right), j=2, \ldots, n$ are suitable functions of $\left[\Gamma_{s}\right]_{11}, \ldots,\left[\Gamma_{s}\right]_{j-1, j-1}$. Therefore, it is sufficient to pick any $\left[\Gamma_{s}\right]_{11}>0$ and for each increasing $j \geqslant 2$ select $\left[\Gamma_{s}\right]_{j j}>0$ such that $\mathfrak{T}^{(j)}<0$.
}

$$
\begin{aligned}
& \mathfrak{T}:=-2 \Gamma_{s}+\left[I-A^{T} \Gamma_{s}\right]^{-1}\left[A+A^{T} \Gamma_{s}^{2}\right] \\
& +\left[A+A^{T} \Gamma_{s}^{2}\right]^{T}\left[I-A^{T} \Gamma_{s}\right]^{-T}<0 .
\end{aligned}
$$

(II) Find $c_{s}, \alpha_{s}>0$ such that for all $x^{\circ} \in \mathscr{B}_{s}:=\{z \in$ $\left.\mathbb{R}^{n}:\|z\|^{2} \leqslant 2 c_{s}\right\}$

$$
\mathfrak{R}_{s}\left(x^{\circ}\right):=-2 \Gamma_{s}+\Phi_{s}\left(x^{\circ}\right)+\Phi_{s}^{T}\left(x^{\circ}\right) \leqslant-\alpha_{s} I,
$$

where

$$
\begin{aligned}
& \Phi_{s}\left(x^{\circ}\right):=\left[I-A^{T} \Gamma_{s}\right]^{-1}\left[A+A^{T} \Gamma_{s}^{2}+\Phi_{s}^{\mathfrak{X}}\left(x^{\circ}\right)\right] \\
& +\left[A+A^{T} \Gamma_{s}^{2}+\Phi_{s}^{\mathfrak{X}}\left(x^{\circ}\right)\right]^{T}\left[I-A^{T} \Gamma_{s}\right]^{-T},
\end{aligned}
$$

and $\Phi_{s}^{\mathfrak{X}}$ is any continuous function such that for all $z:\|z\| \leqslant\left\|x^{\circ}\right\|$

$$
\begin{aligned}
& \Phi_{s}^{\mathfrak{X}}\left(x^{\circ}\right) \\
& \geqslant \Phi\left(A^{T} \Gamma_{s} z, x^{\circ}\right)+\Phi\left(A^{T} \Gamma_{s} z,-A^{T} \Gamma_{s} z\right) A^{T} \Gamma_{s} .
\end{aligned}
$$

The number $c_{s}$ always exists on account of step (I) and since $\Phi(0,0)=0$ (if $\Phi(0,0) \neq 0$, in order to find $\mathscr{B}_{s}$ is enough to satisfy (17)).

(III) Pick $\varepsilon \geqslant 1$ such that

$$
\begin{aligned}
& \left(\sum_{j=1}^{n}\right)\left(\left(\sum_{l=1}^{j}\right)\left[\left(I-A^{T} \Gamma_{s}\right)^{-1}\right]_{j l} \varepsilon^{-\mathfrak{r}_{l}}\left|x_{l}\right|\right)^{2} \leqslant 2 c_{s} \\
& \forall x \in \mathscr{R} .
\end{aligned}
$$

An estimate of the region of attraction $\mathscr{C} \supset \mathscr{R}$ of (14)-(16) as a function of $\varepsilon$ and $c$ can be given as follows. Let $d \in \mathbb{R}_{>}^{n}$ be such that $\|(I-$ $\left.A^{T} \Gamma_{s}\right)^{-1} d \|^{2} \leqslant 2 c_{s}$. Therefore, $\mathscr{C}=\left\{x \in \mathbb{R}^{n}:\left|x_{l}\right| \leqslant\right.$ $\left.d_{l} \varepsilon^{\mathfrak{r}_{l}}, l=1, \ldots, n\right\}$ for each $\varepsilon>1$.

\subsection{PROOF OF THE MAIN RESULT}

Proof of theorem IV.1. Let $I \in \mathbb{R}^{n \times n}$ be the identity matrix and $G_{s} \in \mathbb{R}^{n \times n}$ a diagonal positive definite matrix and identify (whenever necessary) $G_{s}, I$ and $A$ with linear maps $G_{s}, I \in \mathbf{D}^{1}\left(\mathbb{R}^{n}\right)$ and $A \in \mathbf{C}^{1}\left(\mathbb{R}^{n}, \mathbb{R}^{n}\right)$. In particular, select $G_{s}$ in such a way that it is i.g.h. with quadruple $\left(\mathfrak{r}, \mathfrak{f}, \mathfrak{f}, \Gamma_{s}\right)$ with $\Gamma_{s} \in \mathbb{R}^{n \times n}$ is a diagonal positive definite matrix chosen as pointed out in (18) (constructive step (I)).

Let $\mathfrak{X}_{s}:=I-A^{T} G_{s}$. Perform the change of coordinates

$$
\hat{x}=\mathfrak{X}_{s}^{-1} x
$$

After some calculations the closed-loop system (14)(16) in the new coordinates is

$$
\dot{\hat{\mathbf{x}}}=-\mathbf{G}_{\mathbf{s}} \hat{\mathbf{x}}+\rho(\hat{\mathbf{x}})
$$


with

$$
\rho(\hat{x})=\left[I-A^{T} G_{s}\right]^{-1}\left[\left(A-A^{T} G_{s}^{2}\right) \hat{x}+\phi\left(\mathfrak{X}_{s} \hat{x}\right)\right](25)
$$

By lemma VIII.2 $\rho$ is g.h.u.b. with quadruple $\left(\mathfrak{r}, \mathfrak{f}, \mathfrak{f}, \Phi_{s}\right)$ where $\Phi_{s}$ is defined in (20). With $V_{s}(\hat{x}):=$ $\frac{1}{2}\left\|\varepsilon^{-\mathfrak{r}} \diamond \hat{x}\right\|^{2}$ and $x^{\circ}:=\varepsilon^{-\mathfrak{r}} \diamond \hat{x}$ it follows that

$$
\begin{aligned}
& \frac{\partial V_{s}}{\partial \hat{x}}(\hat{x})\left\{-G_{s} \hat{x}+\rho(\hat{x})\right\} \\
& =-\left(\varepsilon^{-2 \mathfrak{r}} \diamond \hat{x}\right)^{T} G_{s} \hat{x}+\left(\varepsilon^{-2 \mathfrak{r}} \diamond \hat{x}\right)^{T} \rho(\hat{x}) \\
& =-\left(\varepsilon^{-\mathfrak{r}} \diamond x^{\circ}\right)^{T} G_{s}\left(\varepsilon^{\mathfrak{r}} \diamond x^{\circ}\right)+\left(\varepsilon^{-\mathfrak{r}} \diamond x^{\circ}\right)^{T} \rho\left(\varepsilon^{\mathfrak{r}} \diamond x^{\circ}\right) \\
& \leqslant-\left(\varepsilon^{\mathfrak{f}} \diamond x^{\circ}\right)^{T} \Gamma_{I}\left(\varepsilon^{\mathfrak{f}} \diamond x^{\circ}\right)+\left(\varepsilon^{\mathfrak{f}} \diamond x^{\circ}\right)^{T} \Phi_{s}\left(x^{\circ}\right)\left(\varepsilon^{\mathfrak{f}} \diamond x^{\circ}\right) \\
& \leqslant-\left(\varepsilon^{\mathfrak{f}} \diamond x^{\circ}\right)^{T}\left[\Gamma_{I}+\Phi_{s}\left(x^{\circ}\right)\right]\left(\varepsilon^{\mathfrak{f}} \diamond x^{\circ}\right) \\
& =\frac{1}{2}\left(\varepsilon^{\mathfrak{f}} \diamond x^{\circ}\right)^{T} \mathfrak{R}_{s}\left(x^{\circ}\right)\left(\varepsilon^{\mathfrak{f}} \diamond x^{\circ}\right)
\end{aligned}
$$

By constructive step (II)

$$
\frac{\partial V_{s}}{\partial \hat{x}}(\hat{x})\left\{-G_{s} \hat{x}+\rho(\hat{x})\right\} \leqslant-\alpha_{s}\left\|\varepsilon^{\mathfrak{f}-\mathfrak{r}} \diamond \hat{x}\right\|^{2}(27)
$$

for all $\hat{x}: V_{s}(\hat{x}) \leqslant c_{s}$. With $W_{s}(x):=V_{s}\left(\mathfrak{X}_{s}^{-1} x\right)$ it follows that

$$
\begin{aligned}
& \left.\dot{W}_{s}\right|_{(14)-(16)}=\frac{\partial W_{s}}{\partial x}(x)\left[A x+B B^{T} \mathfrak{U}^{(n)}(x)\right] \\
& \leqslant-\alpha_{s}\left\|\varepsilon^{\mathfrak{f}-\mathfrak{r}} \diamond \mathfrak{X}_{s}^{-1} x\right\|^{2}
\end{aligned}
$$

for all $x: W_{s}(x) \leqslant c_{s}$. Since $W_{s}$ is positive definite on $\mathbb{R}^{n}$, this proves that for each $\varepsilon \geqslant 1$ the equilibrium $\mathbf{x}=0$ of the closed-loop system (14)-(16) is locally asymptotically stable with region of attraction containing $\left\{z \in \mathbb{R}^{n}: W_{s}(z) \leqslant c_{s}\right\}$. To complete the proof of the theorem, we only need show that for each compact set $\mathscr{R} \subset \mathbb{R}^{n}$ we can pick $\varepsilon \geqslant 1$ in such a way that $\mathscr{R} \subset$ $\left\{z \in \mathbb{R}^{n}: W_{s}(z) \leqslant c_{s}\right\}$ (viz. the given compact set $\mathscr{R}$ is contained in the region of attraction of the equilibrium $\mathbf{x}=0$ of (14)-(16)). We will do this by showing that if $\varepsilon$ is selected as pointed out in (22) (constructive step (III)) then $\max _{x \in \mathscr{R}} W_{s}(x) \leqslant c_{s}$. Indeed, since $\mathfrak{f}_{1} \leqslant$ $\mathfrak{f}_{2} \leqslant \cdots \leqslant \mathfrak{f}_{n}$ is an increasing sequence by $(\mathbf{S})$, we have on account of (22)

$$
\begin{aligned}
& \max _{x \in \mathscr{R}} W_{s}(x) \leqslant \frac{1}{2} \max _{x \in \mathscr{R}}\left\|\varepsilon^{-\mathfrak{r}} \diamond \mathfrak{X}_{s}^{-1}(x)\right\|^{2} \\
& \leqslant \frac{1}{2}\left(\sum_{j=1}^{n}\right)\left(\left(\sum_{l=1}^{j}\right)\left[X_{s}^{-1}\right]_{j l} \varepsilon^{-\mathfrak{r}_{l}} \max _{x \in \mathscr{R}}\left|x_{l}\right|\right)^{2} \\
& =\frac{1}{2}\left(\sum_{j=1}^{n}\right)\left(\left(\sum_{l=1}^{j}\right)\left[\left(I-A^{T} \Gamma_{s}\right)^{-1}\right]_{j l} \varepsilon^{-\mathfrak{r}_{l}} \max _{x \in \mathscr{R}}\left|x_{l}\right|\right)^{2} \\
& =\leqslant c_{s} . \square
\end{aligned}
$$

\section{OBSERVER DESIGN}

\subsection{STATEMENT OF THE MAIN RESULT}

Consider the system

$$
\dot{\mathbf{x}}=A \mathbf{x}+\phi(\mathbf{x}), \mathbf{y}=C \mathbf{x}+\psi(\mathbf{x})
$$

with state $x \in \mathbb{R}^{n}$, output $y \in \mathbb{R}, A$ in Brunowski canonical form with

$$
C=\left(\begin{array}{llll}
1 & 0 & \cdots & 0
\end{array}\right)
$$

$\phi \in \mathbf{C}^{0}\left(\mathbb{R}^{n}, \mathbb{R}^{n}\right), \quad \phi(0)=0, \quad$ and $\quad \psi \in \mathbf{C}^{0}\left(\mathbb{R}^{n}, \mathbb{R}\right)$, $\psi(0)=0$. Also, let $\mathbf{x}\left(\cdot, x_{0}\right)$ (or simply by $\mathbf{x}$ when there is no ambiguity) and $\mathbf{y}\left(\cdot, x_{0}\right)$ (or simply by $\mathbf{y}$ ) denote the state and, respectively, the output trajectory of (29) ensuing from $x_{0}$. We say that $\sigma(h, \cdot) \in \mathbf{D}^{0}\left(\mathbb{R}^{n}\right)$ is a saturation function with levels $h=\left(\begin{array}{lll}h_{1} & \cdots & h_{n}\end{array}\right)^{T}$, $h_{1}, \ldots, h_{n}>0$, if for each $i=1, \ldots, n, \sigma_{i}\left(h, s_{i}\right)=s_{i}$ for all $s:\left|s_{i}\right| \leqslant h_{i}$ and $\sigma_{i}\left(h, s_{i}\right)=\operatorname{sign}\left(s_{i}\right) h_{i}$ for all $s:\left|s_{i}\right|>h_{i}\left(\operatorname{sign}\left(s_{i}\right)\right.$ is 1 if $s_{i}>0$ and -1 if $\left.s_{i}<0\right)$.

Theorem V.1 Assume that

(O1) (incremental homogeneity) $C^{T} \psi$ and $\phi$ are i.g.h.u.b. with quadruple $\left(\mathfrak{r},-\mathfrak{g}, \mathfrak{g}, C^{T} \Psi\right)$ and, respectively, $(\mathfrak{r}, \hat{\mathfrak{g}}, \mathfrak{g}, \Phi)$ with $\Phi(0,0)=0, \Psi(0,0)=0$ and $\hat{\mathfrak{g}}_{n}:=\mathfrak{g}_{n}, \quad \hat{\mathfrak{g}}_{j}:=\mathfrak{r}_{j+1}-\mathfrak{r}_{j}-\mathfrak{g}_{j+1}, \quad j=1, \ldots, n-1$, such that $2 \mathfrak{g}_{j+1}-\mathfrak{g}_{j} \leqslant \hat{\mathfrak{g}}_{j} \leqslant \mathfrak{g}_{j}$,

(O2) (boundedness of state trajectories) there exist two compact sets $\mathscr{R}, \mathscr{C} \subset \mathbb{R}^{n}$ such that $\mathbf{x}\left(t, x_{0}\right) \in \mathscr{C}$ for all $t \geqslant 0$ and for all $x_{0} \in \mathscr{R}$.

There exist $K, c_{o}>0$ and a diagonal positive definite $\Gamma_{o} \in \mathbb{R}^{n \times n}$ such that the solution $\mathbf{x}\left(\cdot, x_{0}\right)$, $\xi\left(\cdot, x_{0}\right)$ of (29) coupled with

$$
\begin{aligned}
& \dot{\xi}=A \xi+\phi\left(\sigma\left(c_{o} \varepsilon^{\mathfrak{r}}, \boldsymbol{\xi}\right)\right) \\
& +K^{(n)}\left(\mathbf{y}-C \xi-\psi\left(\sigma\left(c_{o} \varepsilon^{\mathfrak{r}}, \boldsymbol{\xi}\right)\right)\right), \boldsymbol{\xi}\left(0, x_{0}\right)=0,
\end{aligned}
$$

where

$$
\begin{aligned}
& K^{(i)}:=\varepsilon^{2 \mathfrak{g}} \diamond\left\{K C^{T}+A^{T} \Gamma_{o} K^{(i-1)}\right\}, \\
& i=1, \ldots, n, K^{(0)}:=0,
\end{aligned}
$$

are defined and bounded over $[0,+\infty)$ for all $x_{0} \in$ $\mathscr{R}$. Moreover, $\lim _{t \rightarrow+\infty}\left\|\mathbf{x}\left(t, x_{0}\right)-\xi\left(t, x_{0}\right)\right\|=0$ for all $x_{0} \in \mathscr{R}$.

Remark V.1 Assumption (02) is restrictive even for linear system (29). However, theorem V.1 is per se an interesting result on the observer design for systems with bounded state trajectories. $\square$ 
Remark V.2 Note that $\Phi(0,0)=0$ and $\Psi(0,0)=0$ are required. This is the simplest assumption which guarantees that the linear approximation of (29) around the origin is observable. The minimal requirement for this is

$$
\begin{aligned}
& -2\left(K C^{T} C+A^{T} \Gamma_{o} A\right)+\left(2\left(I+A^{T} \Gamma_{o}\right) \Phi(0,0)\right. \\
& \left.+2 K C^{T} \Psi(0,0)+A+A^{T} \Gamma_{o}^{2}\right)\left(I-A^{T} \Gamma_{o}\right)^{-1} \\
& +\left(I-A^{T} \Gamma_{o}\right)^{-T}\left(2\left(I+A^{T} \Gamma_{o}\right) \Phi(0,0)\right. \\
& \left.+2 K C^{T} \Psi(0,0)+A+A^{T} \Gamma_{o}^{2}\right)^{T}<0
\end{aligned}
$$

which is always satisfied by some positive definite diagonal $\Gamma_{o}$ for either strict lower triangular or strict upper triangular $\Phi(0,0)$ and $C^{T} \Psi(0,0)$. $\square$

Remark V.3 The observer gain matrix $K^{(n)} C$ is homogeneous (in the classical sense) only if $\mathfrak{g}_{j}=\mathfrak{g}_{0}$ for all $j=1, \ldots, n$, which corresponds to the choice $\mathfrak{r}_{j+1}-\mathfrak{r}_{j}=2 \mathfrak{g}_{0}$ in (O1). Therefore, the gaps between each pair of consecutive weights are all equal to $2 \mathfrak{g}_{0}$. Under this restriction, (A2) simply says that $\phi$ (resp. $\psi$ ) is homogeneous (in the upper bound) with degree $2 \mathfrak{g}_{0}$ (resp. 0) and weights $\mathfrak{r}$. Under this regard, it is important to say that the saturation function $\sigma$ is a crucial design issue only when $\phi$ and $\psi$ are not homogeneous in the classical sense and, moreover, the saturation levels are directly proportional to the maximal gap between two consecutive weights. $\square$

\subsection{CONSTRUCTIVE PROCEDURE FOR THE OBSERVER (30)}

The construction of the state observer (30) is accomplished according to the following steps:

(IV) Find a diagonal positive definite $\Gamma_{o} \in \mathbb{R}^{n \times n}$ and $K>0$ such that ${ }^{\S}$

$$
\begin{aligned}
& \mathfrak{S}:=-2\left(K C^{T} C+A^{T} \Gamma_{o} A\right) \\
& +\left(A+A^{T} \Gamma_{o}^{2}\right)\left(I-A^{T} \Gamma_{o}\right)^{-1} \\
& +\left(I-A^{T} \Gamma_{o}\right)^{-T}\left(A+A^{T} \Gamma_{o}^{2}\right)^{T}<0 .
\end{aligned}
$$

\footnotetext{
§Inequality (33) can be solved for some $K$ and $\Gamma_{o}$ on account of the fact that $\mathfrak{S}$ can be defined recursively as $\mathfrak{S}=\mathfrak{S}^{(n)}$ where

$\mathfrak{S}^{(j)}:=\left[\begin{array}{c|c}-2\left(\left[\Gamma_{o}\right]_{n-j, n-j}-\left[\Gamma_{o}\right]_{n-j+1, n-j+1}\right) & \mathfrak{M}^{(j-1)} \\ \hline\left(\mathfrak{M}^{(j-1)}\right)^{T} & \mathfrak{S}^{(j-1)}\end{array}\right]$,

$\mathfrak{S}^{(1)}:=-2\left[\Gamma_{o}\right]_{n-1, n-1},\left[\Gamma_{o}\right]_{00}:=K$,

and $\mathfrak{M}^{(j-1)} \in \mathbf{C}^{0}\left(\mathbb{R}^{n-j}, \mathbb{R}\right), j=2, \ldots, n$, are suitable functions of $\left[\Gamma_{s}\right]_{n-j+1, n-j+1}, \ldots,\left[\Gamma_{o}\right]_{n-1, n-1}$. Therefore, it is sufficient to pick any $\left[\Gamma_{o}\right]_{n-1, n-1}>0$ and for each increasing $j=2, \ldots, n$ select $\left[\Gamma_{o}\right]_{n-j, n-j}>0$ such that $\mathfrak{S}^{(j)}<0$.
}

(V) Find $c_{o}, \alpha_{o}>0$ such that

$\mathfrak{R}_{o}:=-2\left(K C^{T} C+A^{T} \Gamma_{o} A\right)+\Omega_{o}+\Omega_{o}^{T} \leqslant-\alpha_{o}$

where $\Omega_{o}$ is a matrix such that for all $z:\|z\|,\|w\| \leqslant n c_{o}$

$$
\begin{aligned}
& \Omega_{o} \geqslant\left(2\left(I+A^{T} \Gamma_{o}\right) \Phi(z, w)\right. \\
& \left.+2 K C^{T} \Psi(z, w)+A+A^{T} \Gamma_{o}^{2}\right)\left(I-A^{T} \Gamma_{o}\right)^{-1} .
\end{aligned}
$$

The number $c_{o}$ always exists on account of (33) and since $\Phi$ and $\Psi$ are continuous and $\Phi(0,0)=0$ and $\Psi(0,0)=0$ (if either $\Phi(0,0) \neq 0$ or $\Psi(0,0) \neq 0$ for finding $c_{o}$ it is enough to satisfy (32)).

(VI) Pick $\varepsilon \geqslant 1$ such that $\left|x_{i}\right| \leqslant c_{o} \varepsilon^{\mathfrak{r}_{i}}$ for all $i=$ $1, \ldots, n$ and $x \in \mathscr{C}$.

\subsection{PROOF OF THE MAIN RESULT}

Proof of theorem V.1. Let $I \in \mathbb{R}^{n \times n}$ be the identity matrix, $G_{o} \in \mathbb{R}^{n \times n}$ a diagonal positive definite matrix and $H>0$ and identify (whenever necessary) $G_{o}, I, H C^{T} C$ and $A$ with linear maps $G_{o}, I, H C^{T} C \in$ $\mathbf{D}^{1}\left(\mathbb{R}^{n}\right)$. In particular, let $G_{o}$ be i.g.h. with quadruple $\left(\mathfrak{r}, A \mathfrak{g}, A \mathfrak{g}, \Gamma_{o}\right)$ and $H$ be such that $H C^{T} C$ is i.g.h. with quadruple $\left(\mathfrak{r}, \mathfrak{g}, \mathfrak{g}, K C^{T} C\right)$, where $K$ and $\Gamma_{o}$ are chosen as pointed out in (33) (constructive step (IV)). Pick $c_{O}>0$ as pointed out in (32) (constructive steps $(\mathbf{V})$ ).

Let $e:=\xi-x$ be the estimation error and $\mathfrak{X}_{o}:=$ $\left(I-A^{T} G_{o}\right)^{-1}$. Perform the change of coordinates

$$
\hat{e}=\mathfrak{X}_{o}^{-1} e
$$

On account of (O2),

$$
\left.\sigma\left(c_{o} \varepsilon^{\mathfrak{r}}, \mathbf{x}\left(t, x_{0}\right)\right)\right)=\mathbf{x}\left(t, x_{0}\right)
$$

for all $x_{0} \in \mathscr{R}$ and for all $t \geqslant 0$. Using this fact, after some calculations the estimation error system in the new coordinates is

$$
\dot{\hat{\mathbf{e}}}=-\mathbf{K}_{\mathbf{o}} \hat{\mathbf{e}}+\zeta(\hat{\mathbf{e}}, \mathbf{x})
$$

with

$$
\begin{aligned}
& K_{o}=H C^{T} C+A^{T} G_{o} A \\
& \zeta(\hat{e}, x)=-K_{o} C^{T}\left[\psi\left(\sigma\left(c_{o} \varepsilon^{\mathfrak{r}}, \mathfrak{X}_{o} \hat{e}+x\right)\right)\right. \\
& \left.-\psi\left(\sigma\left(c_{o} \varepsilon^{\mathfrak{r}}, x\right)\right)\right] \\
& +\left(-K_{o} A^{T}+I\right)\left[\phi\left(\sigma\left(c_{o} \varepsilon^{\mathfrak{r}}, \mathfrak{X}_{o} \hat{e}+x\right)\right)\right. \\
& \left.-\phi\left(\sigma\left(c_{o} \varepsilon^{\mathfrak{r}}, x\right)\right)\right]+\left[A^{T} G_{o} A A^{T} G_{o}+A\right] \mathfrak{X}_{o} \hat{e}
\end{aligned}
$$

By lemma VIII.3 $K_{o}$ is g.h. with quadruple $\left(\mathfrak{s}, \mathfrak{g}, \mathfrak{g}, K C^{T} C+A^{T} \Gamma_{o} A\right)$ and $\zeta$ is g.h.u.b. with 
quadruple $\left(\mathfrak{s}, \mathfrak{g}, \mathfrak{g}, \Omega_{o}\right)$ where $\Omega_{o}$ is defined as in (35). Let $V_{o}(\hat{e}):=\frac{1}{2}\left\|\varepsilon^{-\mathfrak{s}} \diamond \hat{e}\right\|^{2}$. With $e^{\circ}=\varepsilon^{-\mathfrak{s}} \diamond \hat{e}$ and $x^{\circ}=$ $\varepsilon^{-\mathfrak{s}} \diamond x$ it follows that for all $\hat{e}$

$$
\begin{aligned}
& \frac{\partial V_{o}}{\partial \hat{e}}(\hat{e})\left\{-K_{o} \hat{e}+\zeta(\hat{e}, \xi)\right\} \\
& =-\left(\varepsilon^{-2 \mathfrak{s}} \diamond \hat{e}\right)^{T} K_{o} \hat{e}+\left(\varepsilon^{-2 \mathfrak{s}} \diamond \hat{e}\right)^{T} \zeta(\hat{e}, x) \\
& =-\left(\varepsilon^{-\mathfrak{s}} \diamond e^{\circ}\right)^{T} K_{o}\left(\varepsilon^{\mathfrak{s}} \diamond e^{\circ}\right) \\
& +\left(\varepsilon^{-\mathfrak{s}} \diamond e^{\circ}\right)^{T} \zeta\left(\varepsilon^{\mathfrak{s}} \diamond e^{\circ}, \varepsilon^{\mathfrak{s}} \diamond x^{\circ}\right) \\
& \leqslant-\left(\varepsilon^{\mathfrak{g}} \diamond e^{\circ}\right)^{T}\left(K C^{T} C+A^{T} \Gamma_{o} A\right)\left(\varepsilon^{\mathfrak{g}} \diamond e^{\circ}\right) \\
& +\left(\varepsilon^{\mathfrak{g}} \diamond e^{\circ}\right)^{T} \Omega_{o}\left(\varepsilon^{\mathfrak{g}} \diamond e^{\circ}\right) \\
& \leqslant-\left(\varepsilon^{\mathfrak{g}} \diamond e^{\circ}\right)^{T}\left[K C^{T} C+A^{T} \Gamma_{o} A-\Omega_{o}\right)\left(\varepsilon^{\mathfrak{g}} \diamond e^{\circ}\right) \\
& =\frac{1}{2}\left(\varepsilon^{\mathfrak{g}} \diamond e^{\circ}\right)^{T} \mathfrak{R}_{o}\left(\varepsilon^{\mathfrak{g}} \diamond e^{\circ}\right)
\end{aligned}
$$

From (34) (constructive step (V))

$$
\frac{\partial V_{o}}{\partial \hat{e}}(\hat{e})\left\{-K_{o} \hat{e}+\zeta(\hat{e}, x)\right\} \leqslant-\alpha_{o}\left\|\mathcal{E}^{\mathfrak{g}-\mathfrak{s}} \diamond \hat{e}\right\|^{2}
$$

for all $\hat{e}$. With $W_{o}(e):=V_{o}\left(\mathfrak{X}_{o}^{-1} e\right)$ it follows that

$$
\begin{aligned}
& \left.\dot{W}_{o}\right|_{(29)-(30)}=\frac{\partial W_{o}}{\partial e}(e)\left[A e+\phi \circ \sigma\left(c_{o} \varepsilon^{\mathfrak{r}}, \xi\right)-\phi(x)\right. \\
& \left.+G^{(n)}\left(e-\psi \circ \sigma\left(c_{o} \varepsilon^{\mathfrak{r}}, \xi\right)+\psi(x)\right)\right] \\
& \leqslant-\alpha_{o}\left\|\varepsilon^{\mathfrak{g}-\mathfrak{s}} \diamond \mathfrak{X}_{o}^{-1} e\right\|^{2}
\end{aligned}
$$

Since $W_{o}$ is positive definite on $\mathbb{R}^{n}$ and radially unbounded, this proves that for each $\varepsilon \geqslant 1$ and for each fixed trajectory $\mathbf{x}\left(\cdot, x_{0}\right)$ of (36) with $x_{0} \in \mathscr{R}$, the solution $\xi\left(\cdot, x_{0}\right)$ of (30) coupled with (29) is defined and bounded over $[0,+\infty)$. Moreover, $\lim _{t \rightarrow+\infty} \| \mathbf{x}\left(\cdot, x_{0}\right)-$ $\xi\left(\cdot, x_{0}\right) \|=0$ for all $x_{0} \in \mathscr{R}$.

\section{OUTPUT FEEDBACK STABILIZATION}

In this section we consider

$$
\dot{\mathbf{x}}=A \mathbf{x}+B \mathbf{u}+\phi(\mathbf{x}), \mathbf{y}=C \mathbf{x}+\psi(\mathbf{x}),
$$

with state $x \in \mathbb{R}^{n}$, input $u \in \mathbb{R}$, output $y \in \mathbb{R}, \phi \in$ $\mathbf{C}^{0}\left(\mathbb{R}^{n}, \mathbb{R}^{n}\right), \phi(0)=0$, and $\psi \in \mathbf{C}^{0}\left(\mathbb{R}^{n}, \mathbb{R}\right), \psi(0)=0$. We assume that $(A, B)$ is in Brunowski form and

$$
C=\left(\begin{array}{llll}
1 & 0 & \cdots & 0
\end{array}\right)
$$

The main result of the paper is the following.

Theorem VI.1 Assume (S) and (O1)-(O2) with $\mathfrak{g}>$ $\mathfrak{f}$. For each compact set $\mathscr{R} \subset \mathbb{R}^{n}$ around the origin there exist $K, c_{o}>0$, diagonal positive definite $\Gamma_{s}, \Gamma_{o} \in$ $\mathbb{R}^{n \times n}, \varepsilon \geqslant 1$ and a saturation function $\sigma \in \mathbf{D}^{0}\left(\mathbb{R}^{n}\right)$ with levels $c_{o} \varepsilon^{\mathfrak{r}}$ such that the output feedback controller (30) with

$$
\mathbf{u}=F\left(\sigma\left(c_{o} \varepsilon^{\mathfrak{r}}, \boldsymbol{\xi}\right)\right)
$$

where $F$ is defined in (16), asymptotically stabilize the equilibrium $\mathrm{x}=0$ of (42) with region of attraction containing $\mathscr{R}$.

Remark VI.1 The condition $\mathfrak{g}>\mathfrak{f}$ can be interpreted as a "fast state-recovery" condition for the state observer (30). $\square$

\subsection{CONSTRUCTIVE PROCEDURE FOR THE OUTPUT FEEDBACK CONTROLLER (43)-(30)}

The construction of the output feedback controller (43)-(30) is accomplished by following the constructive steps (I), (II), (IV), selecting $c_{o}$ according to (V) and $\varepsilon \geqslant 1$ in such a way that

$$
\begin{aligned}
\text { (VII) } & \min \left\{c_{s}, \frac{c_{o}^{2}}{2\left\|X_{s}\right\|^{2}}\right\} \geqslant \max _{x_{0} \in \mathscr{R}}\left\{\left\|X_{s}^{-1}\right\|^{2}\left\|\varepsilon^{-\mathfrak{r}} \diamond x_{0}\right\|^{2}\right. \\
+ & \frac{8 c_{o}^{2}\left[\Gamma_{s}\right]_{n n}^{2}\left[\sum_{j=1}^{n} \sum_{l=1}^{n}\left[X_{s}^{-1}\right]_{n j}\left[X_{o}\right]_{j l}\right]^{2}}{\alpha_{s} \alpha_{o}} \\
\cdot & \varepsilon^{2\left(\mathfrak{f}_{n}-\mathfrak{g}_{n}\right)}\left[\ln \left(\frac{\left\|X_{o}^{-1}\right\|^{2}\left\|\varepsilon^{-\mathfrak{r}} \diamond x_{0}\right\|^{2} \varepsilon^{4\left(\mathfrak{g}_{1}-\mathfrak{g}_{n}\right)}}{c_{o}^{2}}\right)\right. \\
+ & 1]\}
\end{aligned}
$$

with $X_{s}:=I+A^{T} \Gamma_{s}, \quad X_{s}^{-1}:=\left(I-A^{T} \Gamma_{s}\right)^{-1}, \quad X_{o}:=$ $\left(I-A^{T} \Gamma_{o}\right)^{-1}, X_{o}^{-1}:=I+A^{T} \Gamma_{o}$ and $\alpha_{s}, \alpha_{o}>0$ such that $\mathfrak{R}_{s} \leqslant-\alpha_{s} I$ and $\mathfrak{R}_{o} \leqslant-\alpha_{o} I, \mathfrak{R}_{s}$ defined in (19) and $\mathfrak{R}_{o}$ in (34). Inequality (VII) can be satisfied on account of $\mathfrak{f}_{n}<\mathfrak{g}_{n}$ which follows from the assumption $\mathfrak{f}<\mathfrak{g}$.

\section{CONCLUSIONS}

We introduced the notion of incremental generalized homogeneity, giving new results on semiglobal stabilization by output feedback and observer design and putting into a unifying framework the stabilization design for triangular (feedback/feedforward) and homogeneous systems. A state feedback controller and an asymptotic state observer are designed separately by dominating the generalized homogeneity degree of the nonlinearities with the degree of the linear approximation of the system and an output feedback controller is obtained according to a certaintyequivalence principle. Future research will be devoted to the global aspects of the stabilization problem. 


\section{APPENDIX}

Lemma VIII.1 If $\sigma(h, \cdot) \in \mathbf{A}^{0}\left(\mathbb{R}^{n}\right)$ is a saturation function with levels $h$, for each $i=1, \ldots, n$ and for all $y, x \in \mathbb{R}$

$$
|\Delta(h, \cdot)(y, x)| \leqslant 2 \sigma_{i}(h,|y-x|) \leqslant 2|y-x| .
$$

Proof: Case A). For all $y, x$ such that $|y| \geqslant h_{i}$ and $|x| \geqslant h_{i}$ we have

$$
\begin{aligned}
& \left|\Delta \sigma_{i}(h, \cdot)(y, x)\right|=0 \leqslant 2 \sigma_{i}(h,|y-x|) \text { if } y x>0, \\
& \left|\Delta \sigma_{i}(h, \cdot)(y, x)\right|=2 h_{i} \leqslant 2 \sigma_{i}(h,|y-x|) \text { if } y x<0 .
\end{aligned}
$$

Case B). For all $y, x$ such that $|y| \geqslant h_{i}$ and $|x| \leqslant h_{i}$

$$
\begin{aligned}
& \left|\Delta \sigma_{i}(h, \cdot)(y, x)\right|=\left|h_{i}-x\right|=h_{i}-|y|+|y-x| \\
& \leqslant|y-x|=\sigma_{i}(|y-x|) \text { if }|y-x| \leqslant h_{i}, \\
& \left|\Delta \sigma_{i}(h, \cdot)(y, x)\right|=\left|h_{i}-x\right| \leqslant 2 h_{i} \\
& =2 \sigma_{i}(|y-x|) \text { if }|y-x| \geqslant h_{i} .
\end{aligned}
$$

Case C). For all $y, x$ such that $|y| \leqslant h_{i}$ and $|x| \geqslant h_{i}$ follow the steps of case B). $\square$

Lemma VIII.2 The function $\rho$, defined in (25), is g.h.u.b. with quadruple $\left(\mathfrak{r}, \mathfrak{f}, \mathfrak{f}, \Phi_{s}\right)$ with $\Phi_{s}$ defined as in (20).

Proof: We break up the proof in several claims.

Claim \#1. $\mathfrak{X}_{s}:=I-A^{T} G_{s}$ (resp. $A^{T} G_{s}$ ) is i.g.h.u.b. with quadruple $\left(\mathfrak{r}, \mathfrak{f},-\mathfrak{f}, X_{s}\right)$ where $X_{s}:=I+$ $A^{T} \Gamma_{s}$ (resp. $A^{T} \Gamma_{s}$ ). By assumption $(\mathbf{S})$ and the shifting rule (P3.1) with $\mathfrak{w}:=\left(I-A^{T} A\right) \mathfrak{r}, A^{T} G_{s}$ is i.g.h.u.b. with quadruple $\left(\mathfrak{r}, A^{T}(I-A) \mathfrak{r}+A^{T} \mathfrak{f}, \mathfrak{f}, A^{T} \Gamma_{s}\right)$. Since $A^{T}(I-A) \mathfrak{r} \leqslant-2 A^{T} \mathfrak{f}$ on account of $(\mathbf{S}), A^{T} G_{s}$ is g.h.u.b. with quadruple $\left(\mathfrak{r},-A^{T} \mathfrak{f}, \mathfrak{f}, A^{T} \Gamma_{s}\right)$. Therefore, by virtue of the shifting rule (P3.1) with $\mathfrak{w}:=-\left(I-A A^{T}\right) \mathfrak{f}, \quad A A^{T} G_{s}$ g.h.u.b. with quadruple $\left(\mathfrak{r}, A \mathfrak{r}-\mathfrak{r}-\mathfrak{f}, \mathfrak{f}, A A^{T} \Gamma_{s}\right)$. Since $A A^{T} \Gamma_{s}$ is diagonal, by the mixing property (P1) with $\mathfrak{d}^{\prime}:=A \mathfrak{r}-$ $\mathfrak{r}+\mathfrak{f}$ and $\mathfrak{h}^{\prime}:=-\mathfrak{f}, A A^{T} G_{s}$ g.h.u.b. with quadruple $\left(\mathfrak{r}, A \mathfrak{r}-\mathfrak{r}+\mathfrak{f},-\mathfrak{f}, A A^{T} \Gamma_{s}\right)$. Applying once more the shifting rule $\left(\right.$ P3.1) with $\mathfrak{w}:=\left(I-A^{T} A\right)(\mathfrak{r}+$ f), $A^{T} A A^{T} G_{s}=A^{T} G_{s}$ is g.h.u.b. with quadruple $\left(\mathfrak{r}, A^{T} \mathfrak{f}+\left(I-A^{T} A\right) \mathfrak{f},-\mathfrak{f}, A^{T} \Gamma_{s}\right)$. Since $A^{T} \mathfrak{f}+(I-$ $\left.A^{T} A\right) \mathfrak{f} \leqslant A^{T} A \mathfrak{f}+\left(I-A^{T} A\right) \mathfrak{f}=\mathfrak{f}$ on account of $(\mathbf{S})$, it follows that $A^{T} G_{s}$ is g.h.u.b. with quadruple $\left(\mathfrak{r}, \mathfrak{f},-\mathfrak{f}, A^{T} \Gamma_{s}\right)$ and, therefore, $\mathfrak{X}_{s}:=I-A^{T} G_{s}$ is g.h.u.b. with quadruple $\left(\mathfrak{r}, \mathfrak{f},-\mathfrak{f}, X_{s}\right)$ with $X_{s}:=I+$ $A^{T} \Gamma_{s}$.

Claim \#2. $A^{T} G_{s}$ is g.h.u.b. with quadruple $\left(\mathfrak{r}, 0,0, A^{T} \Gamma_{s}\right)$. On account of $(\mathbf{S})$ and since $\Gamma_{s}$ is diagonal, by the mixing property $(\mathbf{P 1})$ with $\mathfrak{d}^{\prime}:=2 \mathfrak{f}$ and $\mathfrak{h}^{\prime}:=0, G_{s}$ is g.h.u.b. with quadruple $\left(\mathfrak{r}, 2 \mathfrak{f}, 0, \Gamma_{s}\right)$. Therefore, by the shifting rule (P3.1) with $\mathfrak{w}:=(I-$ $\left.A^{T} A\right) \mathfrak{r}, A^{T} G_{s}$ is g.h.u.b. with quadruple $\left(\mathfrak{r}, 2 A^{T} \mathfrak{f}-\right.$ $\left.A^{T}(A-I) \mathfrak{r}, 0, A^{T} \Gamma_{s}\right)$. Since $2 A^{T} \mathfrak{f} \leqslant A^{T}(A-I) \mathfrak{r}$ by (S), $A^{T} G_{s}$ is g.h.u.b. with quadruple $\left(\mathfrak{r}, 0,0, A^{T} \Gamma_{s}\right)$.

Claim \#3. $\phi \circ \mathfrak{X}_{I}+A-A^{T} G_{s}^{2}$ is g.h.u.b. with quadruple $\quad\left(\mathfrak{r}, \mathfrak{f}, \mathfrak{f}, \Phi_{s}^{\mathfrak{X}}+A+A^{T} \Gamma_{s}^{2}\right), \quad \Phi_{s}^{\mathfrak{X}} \quad$ defined in (21). By (S) and the shifting property (P2.1) with $\mathfrak{w}:=\mathfrak{r}-\mathfrak{f}, A^{T} G_{s}$ is g.h.u.b. with quadruple $\left(\mathfrak{r},-\left(I-A^{T} A\right) \mathfrak{f}-A^{T}(A \mathfrak{r}-\mathfrak{r}-\mathfrak{f}), \mathfrak{f}, A^{T} \Gamma_{s}\right)$.

On account of (S), claim \#2 and using the chaining rule $(\mathbf{P 2}), \phi \circ\left(-A^{T} G_{s}\right)$ is g.h.u.b. with quadruple $\left(\mathfrak{r}, \mathfrak{f}, \mathfrak{f}, \Phi_{2}^{X}\right)$ where $\Phi_{2}^{X}(\hat{x}):=$ $\max _{z:\left|z_{j}\right| \leqslant\left|\hat{x}_{j}\right|} \Phi\left(A^{T} \Gamma_{s} z,-A^{T} \Gamma_{s} z\right) A^{T} \Gamma_{s}$. Also on account of $(\mathbf{S})$, claim $\# 2$ and using the definition of i.g.h.u.b. degree, $\phi \circ \mathfrak{X}_{s}-\phi \circ\left(-A^{T} G_{s}\right)$ is g.h.u.b. with quadruple $\left(\mathfrak{r}, \mathfrak{f}, \mathfrak{f}, \Phi_{1}^{\mathfrak{X}}\right)$ where $\Phi_{1}^{\mathfrak{X}}(\hat{x}):=\max _{z:\left|z_{j}\right| \leqslant\left|\hat{x}_{j}\right|} \Phi\left(A^{T} \Gamma_{s} z, \hat{x}\right)$. Therefore, $\phi \circ \mathfrak{X}_{s}$ is g.h.u.b. with quadruple $\left(\mathfrak{r}, \mathfrak{f}, \mathfrak{f}, \Phi_{s}^{\mathfrak{X}}\right)$ where $\Phi_{s}^{\mathfrak{X}}:=\Phi_{1}^{\mathfrak{X}}+\Phi_{2}^{\mathfrak{X}}$.

By virtue of claim \#1, the chaining rule $(\mathbf{P 2})$ and on account of $(\mathbf{S})$, we conclude also that $A^{T} G_{s}^{2}$ is g.h.u.b. with quadruple $\left(\mathfrak{r}, \mathfrak{f}, \mathfrak{f}, A^{T} \Gamma_{s}^{2}\right)$. Moreover, since $A^{T} A$ is g.h.u.b. with quadruple $\left(\mathfrak{r}, 0,0, A^{T} A\right)$ and $A^{T} A$ is diagonal, by the mixing property (P1) with $\mathfrak{d}^{\prime}:=-\mathfrak{f}$ and $\mathfrak{h}^{\prime}:=\mathfrak{f}, A^{T} A$ is g.h.u.b. with quadruple $\left(\mathfrak{r},-\mathfrak{f}, \mathfrak{f}, A^{T} A\right)$. By the shifting rule (P3.1) with $\mathfrak{w}:=$ $\mathfrak{r}+\mathfrak{f}$ and on account of $(\mathbf{S}), A A^{T} A=A$ is g.h.u.b. with quadruple $((\mathfrak{r}, \mathfrak{f}, \mathfrak{f}), A)$. This concludes the proof of claim \#3.

Claim \#4. $\mathfrak{X}_{s}^{-1}:=\left(I-A^{T} G_{s}\right)^{-1}$ is g.h.u.b. with quadruple $\quad\left(\mathfrak{r}, \mathfrak{f},-\mathfrak{f}, X^{-1}\right), \quad X^{-1}:=\left[I-A^{T} \Gamma_{I}\right]^{-1}$. Note that by definition of $\mathfrak{X}_{s}$ for all $x$ we have $\quad \mathfrak{X}_{s}^{-1}(x)=x+A^{T} G_{s} \mathfrak{X}_{s}^{-1}(x) . \quad$ Therefore, $\mathfrak{X}_{s}^{-1}=\sum_{j=0}^{n-1}\left(A^{T} G_{s}\right)^{j}$. Clearly, $\quad\left(A^{T} G_{s}\right)^{0}=I \quad$ is g.h.u.b. with quadruple $(\mathfrak{r}, 0,0, I)$. Therefore, since $I$ is diagonal and invoking the mixing property $(\mathbf{P 1})$ with $\mathfrak{d}^{\prime}:=\mathfrak{f}$ and $\mathfrak{h}^{\prime}:=-\mathfrak{f},\left(A^{T} G_{s}\right)^{0}$ is g.h.u.b. with quadruple $(\mathfrak{r}, \mathfrak{f},-\mathfrak{f}, I)$. Assume by induction that $\left(A^{T} G_{s}\right)^{j}$ is g.h.u.b. with quadruple $\left(\mathfrak{r}, \mathfrak{f},-\mathfrak{f},\left(A^{T} \Gamma_{s}\right)^{j}\right)$ for all $j=1, \ldots, i$ and for some $i<n-1$. Since $\left(A^{T} G_{s}\right)^{i+1}=\left(A^{T} G_{s}\right)^{i}\left(A^{T} G_{s}\right)$ and both $\left(A^{T} G_{s}\right)^{i}$ and $A^{T} G_{s}$ are g.h.u.b. with quadruple $\left(\mathfrak{r}, \mathfrak{f},-\mathfrak{f},\left(A^{T} \Gamma_{s}\right)^{i}\right)$ and, respectively, $\left(\mathfrak{r}, \mathfrak{f},-\mathfrak{f}, A^{T} \Gamma_{s}\right)$, by claim \#2 and using the chaining rule $(\mathbf{P 2}),\left(A^{T} G_{s}\right)^{i+1}$ is g.h.u.b. with quadruple $\left(\mathfrak{r}, \mathfrak{f},-\mathfrak{f},\left(A^{T} \Gamma_{s}\right)^{i+1}\right)$. By induction it follows that $\left(A^{T} G_{s}\right)^{j}$ for all $j=0, \ldots, n-1$ is g.h.u.b. with quadruple $\left(\mathfrak{r}, \mathfrak{f},-\mathfrak{f},\left(A^{T} \Gamma_{s}\right)^{j}\right)$. Therefore, $\mathfrak{X}_{s}^{-1}=\sum_{j=0}^{n-1}\left(A^{T} G_{s}\right)^{j}$ is g.h.u.b. with quadruple $\quad\left(\mathfrak{r}, \mathfrak{f},-\mathfrak{f}, X_{s}^{-1}\right), \quad X_{s}^{-1}:=\sum_{j=0}^{n-1}\left(A^{T} \Gamma_{s}\right)^{j}=$ $\left[I-A^{T} \Gamma_{I}\right]^{-1}$. 
By claims \#3 and \#4 and using the chaining rule (P2), we conclude that $\phi_{I}:=\left(I-A^{T} G_{s}\right)^{-1}\left[\phi \circ \mathfrak{X}_{I}+\right.$ $\left.A-A^{T} G_{s}^{2}\right]$ is g.h.u.b. with quadruple $\left(\mathfrak{r}, \mathfrak{f}, \mathfrak{f}, \Phi_{s}\right), \Phi_{s}$ defined in (20). This concludes the proof of the lemma. 口

Lemma VIII.3 The functions $K_{o}$ and $\zeta$, defined in (38), are g.h. with quadruple $\left(\mathfrak{s}, \mathfrak{g}, \mathfrak{g}, K C^{T} C+A^{T} \Gamma_{o} A\right)$ and, respectively, g.h.u.b. with quadruple $\left(\mathfrak{s}, \mathfrak{g}, \mathfrak{g}, \Omega_{o}\right)$, $\Omega_{o}$ defined in (35).

Proof: We break up the proof in several claims.

Claim \#1. $\mathfrak{X}_{o}^{-1}$ (resp. $\left.\mathfrak{X}_{o}\right)$ is i.g.h.b. with quadruple $\left(\mathfrak{r},-\mathfrak{g}, \mathfrak{g}, X_{o}^{-1}\right)$ (resp. $\left(\mathfrak{r},-\mathfrak{g}, \mathfrak{g}, X_{o}\right)$ ). This claim follows on account of (O1) using the chaining rule (P2) and the shifting rule (P3.1).

Claim \#2. $K_{o}$ is i.g.h. with quadruple $\left(\mathfrak{s}, \mathfrak{g}, \mathfrak{g}, K C^{T} C+A^{T} \Gamma_{o} A\right)$. By the shifting rules (P3.1) and (P3.2) with $\mathfrak{w}:=\left(I-A^{T} A\right) \mathfrak{g}$ and on account of (O1), $A^{T} G_{O} A$ is i.g.h. with quadruple $\left(\mathfrak{r}, \mathfrak{g}-\left(I-A^{T}\right) \mathfrak{r}, \mathfrak{g}+\left(I-A^{T}\right) \mathfrak{r}, A^{T} \Gamma_{o} A\right)$. Since $A^{T} G_{O} A$ is diagonal, by the mixing property (P1) with $\mathfrak{d}^{\prime}:=\mathfrak{g}$ and $\mathfrak{h}^{\prime}:=\mathfrak{g}, A^{T} G_{o} A$ is i.g.h. with quadruple $\left(\mathfrak{r}, \mathfrak{g}, \mathfrak{g}, A^{T} \Gamma_{o} A\right)$. On account of (O1) $K_{O}$ is i.g.h. with quadruple $\left(\mathfrak{r}, \mathfrak{g}, \mathfrak{g}, K C^{T} C+A^{T} \Gamma_{o} A\right)$ but by the linearity of $K_{o}$ it follows that $K_{o}$ is also i.g.h. with quadruple $\left(\mathfrak{s}, \mathfrak{g}, \mathfrak{g}, K C^{T} C+A^{T} \Gamma_{o} A\right)$.

Claim \#3. $\sigma$ (resp. $\sigma \circ \mathfrak{X}_{o}$ and $\sigma \circ \mathfrak{X}_{o}^{-1}$ ) is i.g.h.u.b. with quadruple $(\mathfrak{s},-\mathfrak{g}, \mathfrak{g}, 2 I) \quad$ (resp. ( $\left.\mathfrak{s},-\mathfrak{g}, \mathfrak{g}, 2 X_{o}\right)$ and $\left.\left(\mathfrak{s},-\mathfrak{g}, \mathfrak{g}, 2 X_{o}^{-1}\right)\right)$. On account of lemma VIII.1 with $h:=c_{o} \varepsilon^{\mathfrak{r}}, \sigma$ is i.g.h.u.b. with quadruple $(\mathfrak{s}, 0,0,2 I)$. By the mixing property (P1) with $\mathfrak{d}^{\prime}:=-\mathfrak{g}$ and $\mathfrak{h}^{\prime}:=\mathfrak{g}, \sigma$ is also i.g.h.u.b. with quadruple $(\mathfrak{s},-\mathfrak{g}, \mathfrak{g}, 2 I)$. Finally, by virtue of the chaining rule (P2) and claim \#1 $\sigma \circ \mathfrak{X}_{o}$ is i.g.h.u.b. with quadruple $\left(\mathfrak{s},-\mathfrak{g}, \mathfrak{g}, 2 X_{o}\right)$.

Claim \#4. $A$ is i.g.h.u.b. with quadruple $(\mathfrak{r}, \mathfrak{g}, \mathfrak{g}, A)$. Note that $I$ is i.g.h.u.b. with quadruple $(\mathfrak{r}, 0,0, I)$, therefore by the mixing property (P1) with $\mathfrak{d}^{\prime}:=-\mathfrak{g}$ and $\mathfrak{h}^{\prime}:=\mathfrak{g}, I$ is also i.g.h.u.b. with quadruple $(\mathfrak{r},-\mathfrak{g}, \mathfrak{g}, I)$. Since $A=A I$, by the shifting rule (P3.1) with $\mathfrak{v}=\mathfrak{r}+\mathfrak{g}, A$ is i.g.h.u.b. with quadruple $\left(\mathfrak{r}, A \mathfrak{r}-A A^{T} \mathfrak{r}-A \mathfrak{g}+\left(I-A A^{T}\right) \mathfrak{g}, \mathfrak{g}, A\right)$. Upon noting that $A \mathfrak{r}-A A^{T} \mathfrak{r}=A A^{T}(A \mathfrak{r}-\mathfrak{r}) \leqslant$ $A A^{T}(A \mathfrak{g}+\mathfrak{g})=A \mathfrak{g}+A A^{T} \mathfrak{g}$ by (A3), $A$ is i.g.h.u.b. with quadruple $(\mathfrak{r}, \mathfrak{g}, \mathfrak{g}, A)$.

Claim \#5. $A^{T} G_{O}$ is i.g.h.u.b. with quadruple $\left(\mathfrak{r},-\mathfrak{g}, \mathfrak{g}, A^{T} \Gamma_{o}\right)$. Since on account of (O1) $G_{o}$ is i.g.h.u.b. with quadruple $\left(\mathfrak{r}, A \mathfrak{g}, A \mathfrak{g}, \Gamma_{o}\right)$ and $\Gamma_{o}$ is diagonal, by the mixing property (P1) with $\mathfrak{d}^{\prime}:=2 A \mathfrak{g}-\mathfrak{g} \quad$ and $\quad \mathfrak{h}^{\prime}:=\mathfrak{g}, \quad G_{O}$ is i.g.h.u.b. with quadruple $\left(\mathfrak{r}, 2 A \mathfrak{g}-\mathfrak{g}, \mathfrak{g}, \Gamma_{o}\right)$. By the shifting rule (P3.1) with $\mathfrak{w}=\left(I-A^{T} A\right)(\mathfrak{r}-\mathfrak{g}), A^{T} G_{o}$ is i.g.h.u.b. with quadruple $\left(\mathfrak{r}, A^{T}(2 A \mathfrak{g}-\mathfrak{g}+\mathfrak{r})-\mathfrak{r}+\left(I-A^{T} A\right)(\mathfrak{r}-\right.$ $\left.\mathfrak{g}), \mathfrak{g}, A^{T} \Gamma_{o}\right)$. On account of (O1) $A^{T}(2 A \mathfrak{g}+\mathfrak{r}) \leqslant$ $A^{T} A \mathfrak{r}-A^{T}(A \mathfrak{g}-\mathfrak{g})$. Therefore, $A^{T} G_{O}$ is i.g.h.u.b. with quadruple $\left(\mathfrak{r},-\mathfrak{g}, \mathfrak{g}, A^{T} \Gamma_{o}\right)$. This ends the proof of claim \#5.

We are ready to prove that $\zeta$ is g.h. with quadruple $\left(\mathfrak{s}, \mathfrak{g}, \mathfrak{g}, \Omega_{o}\right)$ by using the above claims. Since $\sigma$ has saturation levels $c_{o} \varepsilon^{\mathfrak{r}}$ we find out that for each $i, j=$ $1, \ldots, n$

$$
\begin{aligned}
& \sup _{\varepsilon \geqslant 1} \Phi_{i j}\left(\varepsilon^{-\mathfrak{r}} \diamond \sigma\left(c_{o} \varepsilon^{\mathfrak{r}}, \mathfrak{X}_{o}(y)\right), \varepsilon^{-\mathfrak{r}} \diamond \sigma\left(c_{o} \varepsilon^{\mathfrak{r}}, \mathfrak{X}_{o}(x)\right)\right) \\
& \leqslant \max _{\substack{\|z\| \leqslant n c_{o} \\
\|w\| \leqslant n c_{o}}} \Phi_{i j}(z, w):=\Phi_{i j}^{0} .
\end{aligned}
$$

Moreover, on account of claim \#3 and since $\mathfrak{s}-$ $\mathfrak{r}=\mathbb{1} \cdot\left(\mathfrak{g}_{1}-\mathfrak{g}_{n}\right), \sigma \circ \mathfrak{X}_{o}$ is i.g.h.u.b. with quadruple $\left(\mathfrak{r},-\mathfrak{g}, \mathfrak{g}, 2 X_{o}\right)$. Also, on account of (O1) $\phi$ is i.g.h.u.b. with quadruple $(\mathfrak{r}, \mathfrak{g}, \mathfrak{g}, \Phi)$. By virtue of the chaining rule $(\mathbf{P 2})$, it follows that $\phi \circ \sigma\left(c_{o} \varepsilon^{\mathfrak{r}}, \mathfrak{X}_{o}\right)$ is i.g.h.u.b. with quadruple $\left(\mathfrak{r}, \mathfrak{g}, \mathfrak{g}, 2 \Phi^{0} X_{o}\right)$. Therefore, since $\mathfrak{s}-$ $\mathfrak{r}=\mathbb{1} \cdot\left(\mathfrak{g}_{1}-\mathfrak{g}_{n}\right), \phi \circ \sigma\left(c_{o} \varepsilon^{\mathfrak{r}}, \mathfrak{X}_{o}\right)$ is i.g.h.u.b. with quadruple $\left(\mathfrak{s}, \mathfrak{g}, \mathfrak{g}, 2 \Phi^{0} X_{o}\right)$.

Moreover, by claims \#1 and \#4 and the chaining rule (P2), $A \circ \mathfrak{X}_{o}$ is i.g.h.u.b. with quadruple $\left(\mathfrak{s}, \mathfrak{g}, \mathfrak{g}, A X_{o}\right)$ and, since $\mathfrak{s}-\mathfrak{r}=\mathbb{1} \cdot\left(\mathfrak{g}_{1}-\mathfrak{g}_{n}\right)$, is i.g.h.u.b. with quadruple $\left(\mathfrak{s}, \mathfrak{g}, \mathfrak{g}, A X_{o}\right)$. Therefore, $A \mathfrak{X}_{o}+\phi \circ \sigma\left(c_{o} \varepsilon^{\mathfrak{r}}, \mathfrak{X}_{o}\right)$ is i.g.h.u.b. with quadruple $\left(\mathfrak{s}, \mathfrak{g}, \mathfrak{g}, \Phi_{o}\right)$ and $\Phi_{o}:=\left(A+2 \Phi^{0}\right) X_{o}$.

By (O1) and the shifting rule (P3.1) with $\mathfrak{w}=$ $\left(I-A^{T} A\right)(\mathfrak{r}-\mathfrak{g}), A^{T} \phi$ is i.g.h.u.b. with quadruple $\left(\mathfrak{s},-\mathfrak{g}, \mathfrak{g}, A^{T} \Phi\right)$. Using claims $\# 1$ and $\# 5$ and the chaining rule, we conclude that $C^{T} \psi \circ \sigma\left(c_{o} \varepsilon^{\mathfrak{r}}, \mathfrak{X}_{o}\right)+$ $A^{T} \phi \circ \sigma\left(c_{o} \varepsilon^{\mathfrak{r}}, \mathfrak{X}_{o}\right)+G_{o} \mathfrak{X}_{o}$ is i.g.h.u.b. with quadruple $\left(\mathfrak{s},-\mathfrak{g}, \mathfrak{g}, \Psi_{o}\right), \Psi_{o}:=\left[2 C^{T} \Psi^{0}+A^{T}\left(2 \Phi^{0}+\Gamma_{o}\right)\right] X_{o}$ and for each $j=1, \ldots, n$

$$
\Psi_{j}^{0}:=\max _{\substack{\|z\| \leqslant n_{0},\|w\| \leqslant n c_{o}}} \Psi_{j}(z, w) .
$$

Therefore, using claim \#2 and the chaining rule we obtain that $K_{o} \psi_{o}$ is i.g.h.u.b. with quadruple $\left(\mathfrak{s}, \mathfrak{g}, \mathfrak{g},\left(K C^{T} C+A^{T} \Gamma_{o} A\right) \Psi_{o}\right)$. Upon noting that $A C^{T}=0, \quad C C^{T}=1$ and $A^{T} \Gamma_{o} A A^{T}=A^{T} \Gamma_{o}$, we conclude that $\zeta$ is g.h.u.b. with quadruple $\left(\mathfrak{s}, \mathfrak{g}, \mathfrak{g}, \Omega_{o}\right)$ and $\Omega_{o}$ defined in (35).

The fact that $K_{o}$ is g.h. with quadruple $\left(\mathfrak{s}, \mathfrak{g}, \mathfrak{g}, K C^{T} C+A^{T} \Gamma_{o} A\right)$ follows directly from claim \#2. ㅁ

(Proof of theorem VI.1). Select $G_{s}, \Gamma_{s}$ and $c_{s}$ according the constructive steps (I) and (II), $G_{o}, \Gamma_{o}, c_{o}$ and $\varepsilon$ according the constructive steps (IV), (V) and 
(VII). Let $\mathfrak{X}_{s}, \mathfrak{X}_{o}, \hat{x}, V_{s}, W_{s}, \hat{e}, V_{o}, W_{o}$ be as in the proof of theorems IV.1 and V.1. Denote by $\mathbf{x}\left(t, x_{0}\right), \xi\left(t, x_{0}\right)$ (or simply $\mathbf{x}, \xi$ ) the state trajectories of the closed-loop system (42)-(43)-(30) with initial conditions $x_{0}$ and $\xi_{0}:=0$, with $\mathbf{e}\left(t, x_{0}\right):=\xi\left(t, x_{0}\right)-\mathbf{x}\left(t, x_{0}\right)$ (or simply e). In $(\hat{x}, \hat{e})$ coordinates (42)-(43)-(30) reads as

$$
\begin{aligned}
& \dot{\hat{\mathbf{x}}}=-\mathbf{G}_{\mathbf{s}} \hat{\mathbf{x}}+\rho(\hat{\mathbf{x}}) \\
& +B F\left(\sigma\left(c_{o} \varepsilon^{\mathfrak{r}}, \mathfrak{X}_{o}(\hat{\mathbf{e}})+\mathfrak{X}_{s}(\hat{\mathbf{x}})\right)-\mathfrak{X}_{s}(\hat{\mathbf{x}})\right) \\
& \dot{\hat{\mathbf{e}}}=-\mathbf{K}_{\mathbf{o}} \hat{\mathbf{e}}+\zeta(\hat{\mathbf{e}}, \mathbf{x})
\end{aligned}
$$

Following the same steps for the proof of theorem V.1, we obtain the existence of $\alpha_{o}>0$ such that for each $x_{0} \in \mathscr{R}$

$$
\left.\dot{V}_{o}\right|_{(47)} \leqslant-\alpha_{o}\left\|\varepsilon^{\mathfrak{g}-\mathfrak{s}} \diamond \hat{\mathbf{e}}\right\|^{2} .
$$

From (48) and since $\mathfrak{g}_{1} \geqslant \mathfrak{g}_{2} \geqslant \cdots \geqslant \mathfrak{g}_{n}$, it follows that

$$
\begin{aligned}
& V_{o}\left(\hat{\mathbf{e}}\left(t, x_{0}\right)\right) \leqslant V_{o}\left(\hat{\mathbf{e}}\left(0, x_{0}\right)\right) \exp ^{-\alpha_{O} \varepsilon^{2 \mathfrak{g}_{n}} t} \\
& \leqslant\left\|\varepsilon^{-\mathfrak{s}} \diamond \mathfrak{X}_{o}^{-1}\left(x_{0}\right)\right\|^{2} \exp ^{-\alpha_{O} \varepsilon^{2 \mathfrak{g}_{n}} t}
\end{aligned}
$$

for all $t \geqslant 0$ and $x_{0} \in \mathscr{R}$. Therefore, $\hat{\mathbf{e}} \in \mathbf{L}^{\infty}\left(\mathbb{R}_{+}, \mathbb{R}^{n}\right)$ and $\hat{\mathbf{e}}\left(t, x_{0}\right) \rightarrow 0$ as $t \rightarrow+\infty$ if $x_{0} \in \mathscr{R}$.

Note that

Define $\mathscr{C}:=\left\{z \in \mathbb{R}^{n}:\left|z_{i}\right| \leqslant c_{o} \varepsilon^{\mathfrak{r}_{i}}, i=1, \ldots, n\right\}$.

$$
\hat{x}: \mathfrak{X}_{s}(\hat{x}) \in \mathscr{C} \Rightarrow \sigma\left(c_{o} \varepsilon^{\mathfrak{r}}, \mathfrak{X}_{s}(\hat{x})\right)=\mathfrak{X}_{s}(\hat{x})(50)
$$

Using claim \#1 of lemma VIII. 2 and $\mathfrak{f}_{1} \leqslant \mathfrak{f}_{2} \leqslant \cdots \leqslant$ $\mathfrak{f}_{n}$, it follows that

$$
\left\|\varepsilon^{-\mathfrak{r}} \diamond \mathfrak{X}_{s}(\hat{x})\right\|^{2} \leqslant 2\left\|X_{s}\right\|^{2} V_{s}(\hat{x}) \leqslant c_{o}^{2}
$$

for all $\hat{x}: V_{s}(\hat{x}) \leqslant \frac{c_{o}^{2}}{2\left\|X_{s}\right\|^{2}}$, which implies that $V_{s}(\hat{x}) \leqslant$ $\frac{c_{o}^{2}}{2\left\|X_{s}\right\|^{2}} \Rightarrow \mathfrak{X}_{s}(\hat{x}) \in \mathscr{C}$. Therefore,

$$
\hat{x}: V_{s}(\hat{x}) \leqslant \frac{c_{o}^{2}}{2\left\|X_{s}\right\|^{2}} \Rightarrow \sigma\left(c_{o} \varepsilon^{\mathfrak{r}}, \mathfrak{X}_{s}(\hat{x})\right)=\mathfrak{X}_{s}((\mathfrak{D}))
$$

Following the same steps of the proof of theorem IV.1, using claims \#1 of lemmas VIII.2 and VIII.3 and lemma VIII.1 with $h:=c_{o} \varepsilon^{\mathfrak{r}}$, we obtain the existence of $\alpha_{s}>0$ such that

$$
\begin{aligned}
& \left.\dot{V}_{s}\right|_{(46)} \leqslant-\alpha_{s}\left\|\varepsilon^{\mathfrak{f}-\mathfrak{r}} \diamond \hat{x}\right\|^{2}+\frac{\alpha_{s}}{2} \varepsilon^{2\left(\mathfrak{f}_{n}-\mathfrak{r}_{n}\right)} \hat{x}_{n}^{2} \\
& +\frac{8\left[\Gamma_{s}\right]_{n n}^{2}}{\alpha_{s}} \varepsilon^{2 \mathfrak{f}_{n}}\left(\sum_{j=1}^{n}\left[X_{s}^{-1}\right]_{n j} \min \left\{c_{o}, \varepsilon^{-\mathfrak{r}_{j}}\left|\left[\mathfrak{X}_{o}\right]_{j} \hat{e}\right|\right\}\right)^{2} \\
& \leqslant-\frac{\alpha_{s}}{2}\left\|\varepsilon^{\mathfrak{f}-\mathfrak{r}} \diamond \hat{x}\right\|^{2} \\
& +\frac{8\left[\Gamma_{s}\right]_{n n}^{2}\left[\sum_{j=1}^{n} \sum_{l=1}^{n}\left[X_{s}^{-1}\right]_{n j}\left[X_{o}\right]_{j l}\right]^{2}}{\alpha_{s}} \\
& \cdot \varepsilon^{2 \mathfrak{f}_{n}} \min \left\{c_{o}^{2}, \varepsilon^{4\left(\mathfrak{g}_{1}-\mathfrak{g}_{n}\right)} V_{o}(\hat{e})\right\}
\end{aligned}
$$

for all $(\hat{x}, \hat{e}): V_{s}(\hat{x}) \leqslant \min \left\{c_{s}, \frac{c_{o}^{2}}{2\left\|X_{s}\right\|^{2}}\right\}$.

By integrating (52) over $[0, t], t \geqslant 0$, and on account of (49) we obtain

$$
V_{s}\left(\hat{\mathbf{x}}\left(t, x_{0}\right)\right) \leqslant \min \left\{c_{s}, \frac{c_{o}^{2}}{2\left\|X_{s}\right\|^{2}}\right\}
$$

for all $x_{0}$ such that

$$
\begin{aligned}
& \min \left\{c_{s}, \frac{c_{o}^{2}}{2\left\|X_{s}\right\|^{2}}\right\} \geqslant\left\|\varepsilon^{-\mathfrak{r}} \diamond \mathfrak{X}_{s}^{-1}\left(x_{0}\right)\right\|^{2} \\
& +\frac{8 c_{o}^{2}\left[\Gamma_{s}\right]_{n n}^{2}\left[\sum_{j=1}^{n} \sum_{l=1}^{n}\left[X_{s}^{-1}\right]_{n j}\left[X_{o}\right]_{j l}\right]^{2}}{\alpha_{s} \alpha_{o}} . \\
& \cdot \varepsilon^{2\left(\mathfrak{f}_{n}-\mathfrak{g}_{n}\right)}\left[\ln \left(\frac{\left\|\varepsilon^{-\mathfrak{s}} \diamond \mathfrak{X}_{o}^{-1}\left(x_{0}\right)\right\|^{2} \varepsilon^{4\left(\mathfrak{g}_{1}-\mathfrak{g}_{n}\right)}}{c_{o}^{2}}\right)+1\right]
\end{aligned}
$$

By claim \#4 of lemma VIII.2 and claim \#1 of lemma VIII. 3 and since $\mathfrak{f}_{1} \leqslant \mathfrak{f}_{2} \leqslant \cdots \leqslant \mathfrak{f}_{n}$ and $\mathfrak{g}_{n} \leqslant \cdots \leqslant \mathfrak{g}_{1}$, for each $x_{0}$

$$
\begin{aligned}
& \left\|\varepsilon^{-\mathfrak{r}} \diamond \mathfrak{X}_{s}^{-1}\left(x_{0}\right)\right\| \leqslant\left\|X_{s}^{-1}\right\|\left\|\varepsilon^{-\mathfrak{r}} \diamond x_{0}\right\| \\
& \left\|\varepsilon^{-\mathfrak{s}} \diamond \mathfrak{X}_{o}^{-1}\left(x_{0}\right)\right\| \leqslant\left\|X_{o}^{-1}\right\|\left\|\varepsilon^{-\mathfrak{r}} \diamond x_{0}\right\|
\end{aligned}
$$

As a consequence of the constructive step (VII) any $x_{0} \in \mathscr{R}$ satisfies the inequality (54). Therefore, (53) holds for all $x_{0} \in \mathscr{R}$. It follows that $\hat{\mathbf{x}} \in \mathbf{L}^{\infty}\left(\mathbb{R}_{+}, \mathbb{R}^{n}\right)$ for all $x_{0} \in \mathscr{R}$. The $\Omega$-limit set of $\hat{\mathbf{x}}\left(\cdot, x_{0}\right)$ and $\hat{\mathbf{e}}\left(\cdot, x_{0}\right)$ is non-empty, compact and invariant and it is contained in the set of points $\{(\hat{x}, \hat{e}): \hat{e}=0\}$. This, on account of (52), implies that $\hat{\mathbf{x}}\left(t, x_{0}\right), \hat{\mathbf{e}}\left(t, x_{0}\right) \rightarrow 0$ (and therefore $\left.\mathbf{x}\left(t, x_{0}\right), \xi\left(t, x_{0}\right) \rightarrow 0\right)$ as $t \rightarrow+\infty$ if $x_{0} \in \mathscr{R}$. This proves that the output feedback controller (43)-(30) asymptotically stabilize the equilibrium $\mathbf{x}=0$ of (42) with region of attraction containing $\mathscr{R} . \square$

\section{REFERENCES}

1. V. Andrieu, L. Praly, A. Astolfi, Homogeneous approximation, recursive observer design and output feedback, SIAM Journ. Contr. Optim., 47, pp. 1814-1850, 2008.

2. S. Battilotti, Lyapunov-based design of iISS feedforward systems with uncertainty and noisy measurements, SIAM Journ. Contr. Optim., 46, pp. 84-115, 2007.

3. S. Battilotti, Generalized incremental homogeneity, incremental observability and global observer design, IEEE Conference on Decision and Control, Orlando, 2011.

4. S. Battilotti, Incremental non-homogeneity of nonlinear systems, Triennal IFAC World Conference, Milano, 2011. 
5. H. Hermes, Homogeneous coordinates and continuous asymptotically stabilizing feedback controls, Diff. Eq. Stab. Contr., S. Elyadi ed., Lect. Notes in Applied Math., 109, Marcel Dekker, New York, 1991.

6. J.L. Massera, Contributions to stability theory, Ann. Math., 64, 182-206, 1956.

7. C. Qian, Semi-global stabilization of a class of uncertain nonlinear systems by linear output feedback, IEEE Trans. Circ. Syst.-II, 52, 218-222, 2005.

8. J. Polendo, C. Qian, An expanded method to robustly stabilize uncertain nonlinear systems, Communication in Information and Systems, 8, 55-70, 2008.

9. J. Zhai, C. Qian, H. Du, Semi-global output feedback stabilization for a class of uncertain nonlinear systems, Asian Journal of Control, published on line, 3/28/2012. 2008.

10. H. Shim, Y. I. Son, and J. H. Seo, Semi-global observer formulti-output nonlinear systems, Automatica, 37, 10931101, 2001.

11. J. Zhai, C. Qian, H. Ye, Semi-global output feedback stabilization of a generalized class of MIMO nonlinear systems, ASME J. Dyn. Syst., Meas., Control, 133, 064501.1- 064501.5 , 2011.

12. A. Teel, L. Praly, Tools for semi-global stabilization by partial state and output feedback, SIAM Journ. Contr. Optim., 33, 1443-1488, 1995.

13. L. Rosier, Homogeneous Lyapunov function for homogeneous continuous vector field, Syst. Contr. Lett., 19, 467-473, 1998.

14. B. Yang, W. Lin, Semiglobal output feedback of non-uniformly observable and nonsmoothly stabilizable systems, Proc. IEEE CDC, Seville (Spain), 2005, 4207-4212. 